\title{
Generic absoluteness under projective forcing
}

\author{
by \\ Joan Bagaria (Barcelona) and \\ Roger Bosch (Oviedo)
}

\begin{abstract}
We study the preservation of the property of $L(\mathbb{R})$ being a Solovay model under projective ccc forcing extensions. We compute the exact consistency strength of the generic absoluteness of $L(\mathbb{R})$ under forcing with projective ccc partial orderings and, as an application, we build models in which Martin's Axiom holds for $\sum_{n}^{1}$ partial orderings, but it fails for the $\sum_{n+1}^{1}$.
\end{abstract}

1. Introduction. In this paper we continue the systematic study of the preservation of the property of $L(\mathbb{R})$ being a Solovay model under various classes of forcing notions. This work started in [2], where we considered the class of projective absolutely-ccc forcing notions and obtained an exact consistency result for the preservation of the property of $L(\mathbb{R})$ being a Solovay model under this class of forcing extensions. It turned out that the large cardinals involved were the definably Mahlo cardinals, a weak form of Mahlo cardinals that satisfy some definability conditions. As a corollary we obtained the equiconsistency of: (1) there exists a definably-Mahlo cardinal; and (2) $L(\mathbb{R})$-absoluteness for projective absolutely ccc posets.

In [3] we showed that every projective strongly proper forcing notion preserves the property of $L(\mathbb{R})$ being a definably Mahlo Solovay model. Hence, the consistency of $L(\mathbb{R})$-absoluteness under projective strongly proper forcing notions has the existence of a definably Mahlo cardinal as an upper bound. We also proved in [3] that the consistency strength of the preservation of $L(\mathbb{R})$ being a Solovay model under $\sigma$-linked forcing notions is exactly

2000 Mathematics Subject Classification: 03E15, 03E35, 03E50, 03E55.

Key words and phrases: Solovay model, projective ccc forcing extension, generic absoluteness.

Research partially supported by the MEC-FEDER grants BFM2002-03236 and MTM 2005-01025, and the grants 2002SGR-00126 and 2005SGR-00738 of the Generalitat de Catalunya, and PR-01-GE-10 of the Principado de Asturias. 
that of a Mahlo cardinal, in contrast with the general ccc case, for which a weakly compact cardinal is required.

Recall that a Solovay model over $V$ is the $L(\mathbb{R})$ of a model $M \supseteq V$ which has the following properties:

(1) For every $x \in \mathbb{R}, \omega_{1}$ is an inaccessible cardinal in $V[x]$.

(2) Every $x \in \mathbb{R}$ is small-generic over $V$. That is, for some forcing notion $\mathbb{P}$ in $V$ that is countable in $M$, there is, in $M$, a $\mathbb{P}$-generic filter $g$ over $V$ such that $x \in V[g]$.

The reason we call a model with properties (1) and (2) above a Solovay model is the following result of Woodin (see [2]), which says that it is elementarily equivalent to Solovay's model from [10].

Lemma 1.1. Suppose that $V \subseteq M$ are models of (a fragment of) ZFC and $M$ satisfies (1) and (2) above. Then there is a forcing notion $\mathbb{W}$ in $M$ which does not add new reals and creates a generic filter $C$ for the Levy collapse of $\omega_{1}^{M}$ over $V$ such that $M$ and $V[C]$ have the same reals.

Our interest in the preservation of the property of $L(\mathbb{R})$ being a Solovay model under forcing extensions that do not collapse $\omega_{1}$ lies mainly in the fact (Lemma 1.3 below) that it implies a strong form of generic absoluteness for the theory of the reals (see [2]).

Definition 1.2. Let $V$ be a model of ZF. Let $\mathbb{P} \in V$ be a forcing notion and let $\varphi$ be a formula (possibly with parameters in $V$ ). $V$ is $\varphi$-absolute for $\mathbb{P}$ iff

$$
V \models \varphi \quad \text { iff } \quad V^{\mathbb{P}} \models \varphi .
$$

If $\Sigma$ is a set of formulas, $V$ is $\Sigma$-absolute for $\mathbb{P}$ iff for every $\varphi \in \Sigma, V$ is $\varphi$-absolute for $\mathbb{P}$. Given a class $\Gamma$ of posets, $V$ is $\Sigma$-absolute for $\Gamma$ iff for every $\mathbb{P} \in \Gamma, V$ is $\Sigma$-absolute for $\mathbb{P}$ in $V$.

$V$ is $L(\mathbb{R})$-absolute for $\mathbb{P}$ iff there exists an elementary embedding

$$
j: L(\mathbb{R})^{V} \rightarrow L(\mathbb{R})^{V^{\mathbb{P}}}
$$

that fixes all the ordinals (and therefore all the reals). For $\Gamma$ a class of posets, $V$ is $L(\mathbb{R})$-absolute for $\Gamma$ if it is $L(\mathbb{R})$-absolute for every $\mathbb{P}$ in $\Gamma$.

The following lemma is proved in [2].

Lemma 1.3. Suppose that $L(\mathbb{R})^{M}$ and $L(\mathbb{R})^{N}$ are Solovay models over $V$ such that $\mathbb{R}^{M} \subseteq \mathbb{R}^{N}$ and $\omega_{1}^{M}=\omega_{1}^{N}$. Then there exists an elementary embedding $j: L(\mathbb{R})^{M} \rightarrow L(\mathbb{R})^{N}$ which fixes all the ordinals.

Recall that for $\Gamma$ a point-class, a $\Gamma$-poset is a triple $\mathbb{P}=\left\langle P, \leq_{P}, \perp_{P}\right\rangle$, where $\leq_{P}$ is a $\Gamma$-subset of $\omega^{\omega} \times \omega^{\omega}, P=\operatorname{field}\left(\leq_{P}\right),\left\langle P, \leq_{P}\right\rangle$ is a partial order, and $\perp_{P}$ is a $\Gamma$-subset of $\omega^{\omega} \times \omega^{\omega}$ contained in $P \times P$ such that for every $x, y \in P, x \perp_{P} y$ iff $x, y$ are incompatible. $\mathbb{P}$ is a projective poset iff it 
is (isomorphic to) a $\Gamma$-poset for some projective point-class $\Gamma$. Notice that a poset $\mathbb{P}$ is projective iff it is (isomorphic to a poset that is) first-order definable in $H\left(\omega_{1}\right)$, with parameters.

In this paper we consider the class of projective ccc forcing notions. We show that the property of $L(\mathbb{R})$ being a $\Sigma_{n}$-weakly compact Solovay model (see definitions below) is preserved by forcing with $\sum_{n+1}^{1}$ ccc posets, and that the property of $L(\mathbb{R})$ being a definably weakly compact Solovay model is preserved by all projective ccc posets. We give an example of a $\Delta_{3}^{1}$ poset $\mathbb{P}$ with the property $K$, hence ccc, such that $\Sigma_{4}^{1}$ generic absoluteness under forcing with $\mathbb{P}$ implies that $\omega_{1}$ is $\Sigma_{1}$-weakly compact in $L$. A generalization of this example to higher projective levels shows that the consistency strength of $L(\mathbb{R})$-absoluteness under $\sum_{n+1}^{1}$ ccc forcing is exactly the existence of a $\Sigma_{n}$-weakly compact cardinal. Further, the consistency strength of $L(\mathbb{R})$-absoluteness under projective ccc forcing extensions is exactly that of the existence of a definably weakly compact cardinal. In the last section, and as an application of the previous results, we build models in which Martin's axiom holds for $\sum_{n}^{1}$ partial orderings but not for the $\underset{\sim}{\sum_{n+1}^{1}}$.

2. Projective ccc forcing extensions. We will address the question of the preservation of the property of $L(\mathbb{R})$ being a Solovay model under arbitrary projective ccc forcing notions. As we will see, we need to consider a definable form of weakly compact cardinals.

2.1. $\Sigma_{n}$-weakly compact cardinals. Recall that a $\Pi_{1}^{1}$ sentence of the language of set theory is a sentence of the form $\forall X \varphi(X)$, where $\varphi(X)$ is a first-order formula of the language of set theory expanded with the predicate symbol $X$.

Definition 2.1. Let $\kappa$ be a cardinal and $n \in \omega$. Then $\kappa$ is $\Sigma_{n}$-weakly compact $\left(\Sigma_{n}\right.$-w.c., for short) iff $\kappa$ is inaccessible and for every $R \subseteq V_{\kappa}$ which is definable by a $\Sigma_{n}$ formula (with parameters) over $V_{\kappa}$ and every $\Pi_{1}^{1}$ sentence $\Phi$, if

$$
\left\langle V_{\kappa}, \in, R\right\rangle \models \Phi
$$

then there is $\alpha<\kappa$ (equivalently, unboundedly many $\alpha<\kappa$ ) such that

$$
\left\langle V_{\alpha}, \in, R \cap V_{\alpha}\right\rangle \models \Phi .
$$

That is, $\kappa$ reflects $\Pi_{1}^{1}$ sentences with $\Sigma_{n}$ predicates. Moreover, $\kappa$ being $\Pi_{n}$-weakly compact ( $\Pi_{n}$-w.c., for short) is defined analogously by substituting $\Pi_{n}$ for $\Sigma_{n}$ in the definition above. Thus, an inaccessible cardinal $\kappa$ is $\Pi_{n}$-w.c. iff it reflects $\Pi_{1}^{1}$ sentences with $\Pi_{n}$ predicates. An inaccessible cardinal is $\Delta_{n}$-weakly compact ( $\Delta_{n}$-w.c., for short) iff it reflects $\Pi_{1}^{1}$ sentences with $\Delta_{n}$ predicates. 
Definition 2.2 (A. Leshem, [9]). A cardinal $\kappa$ is $\Sigma_{\omega}$-weakly compact ( $\Sigma_{\omega}$-w.c., for short) iff $\kappa$ is $\Sigma_{n}$-w.c. for every $n \in \omega$.

Proposition 2.3. For $\kappa$ an inaccessible cardinal, the following are equivalent:

(1) $\kappa$ is $\Sigma_{n}-w . c$.

(2) $\kappa$ is $\Pi_{n}$-w.c.

(3) $\kappa$ is $\Delta_{n+1}$-w.c.

(4) For every $\Pi_{1}^{1}$ formula $\Phi\left(x_{0}, \ldots, x_{k}\right)$ in the language of set theory and every $a_{0}, \ldots, a_{k} \in V_{\kappa}$, if $V_{\kappa}=\Phi\left(a_{0}, \ldots, a_{k}\right)$, then there is $\lambda \in$ $I_{n}:=\left\{\lambda<\kappa: \lambda\right.$ is inaccessible and $\left.V_{\lambda} \preccurlyeq_{n} V_{\kappa}\right\}$ such that $V_{\lambda} \models$ $\Phi\left(a_{0}, \ldots, a_{k}\right)$.

Proof. $(3) \Rightarrow(1)$ and $(3) \Rightarrow(2)$ are trivial.

$(1) \Rightarrow(2)$ : Suppose that $R \subseteq V_{\kappa}$. For every $\Pi_{1}^{1}$ formula $\Psi$ where $R$ appears as a predicate, let $\widetilde{\Psi}$ be the formula obtained from $\Psi$ by substituting every occurrence of the subformula $R x$, where $x$ is a first order variable, by $\neg R x$. Note that $\widetilde{\Psi}$ is also $\Pi_{1}^{1}$.

It is easily shown, by induction on the complexity of formulas, that for every formula $\Psi$ and every $\alpha$,

$$
\left\langle V_{\alpha}, \in, R \cap V_{\alpha}\right\rangle \models \Psi \quad \text { iff } \quad\left\langle V_{\alpha}, \in, V_{\alpha} \backslash R\right\rangle \models \widetilde{\Psi} .
$$

Suppose now that $R \subseteq V_{\kappa}$ is definable by means of a $\Pi_{n}$ formula over $V_{\kappa}$ and $\Phi$ is a $\Pi_{1}^{1}$ sentence. If $\left\langle V_{\kappa}, \in, R\right\rangle \models \Phi$, then $\left\langle V_{\kappa}, \in, V_{\kappa} \backslash R\right\rangle \models \widetilde{\Phi}$. Since $\kappa$ is $\Sigma_{n}$-w.c., there is $\alpha<\kappa$ such that $\left\langle V_{\alpha}, \in,\left(V_{\kappa} \backslash R\right) \cap V_{\alpha}\right\rangle=\left\langle V_{\alpha}, \in, V_{\alpha} \backslash R\right\rangle \models \widetilde{\Phi}$, and therefore $\left\langle V_{\alpha}, \in, R \cap V_{\alpha}\right\rangle \models \Phi$.

$(2) \Rightarrow(4)$ : Suppose that $\Phi\left(x_{0}, \ldots, x_{k}\right)=\forall X \varphi\left(X, x_{0}, \ldots, x_{k}\right)$ is a $\Pi_{1}^{1}$ formula and $a_{0}, \ldots, a_{k} \in V_{\kappa}$ are such that $V_{\kappa} \models \Phi\left(a_{0}, \ldots, a_{k}\right)$.

Let $\Psi$ be the $\Pi_{1}^{1}$ sentence expressing that $\kappa$ is inaccessible, and let $\sigma$ be the first order sentence saying that the $\Pi_{n}$-club $C_{n}:=\left\{\alpha<\kappa: V_{\alpha} \preccurlyeq n V_{\kappa}\right\}$ is unbounded. Then

$$
\left\langle V_{\kappa}, \in, C_{n}\right\rangle \models \Phi\left(a_{0}, \ldots, a_{k}\right) \wedge \Psi \wedge \sigma .
$$

Since $\kappa$ is $\Pi_{n}$-w.c., there is $\lambda<\kappa$ such that

$$
\left\langle V_{\lambda}, \in, C_{n} \cap V_{\lambda}\right\rangle \models \Phi\left(a_{0}, \ldots, a_{k}\right) \wedge \Psi \wedge \sigma .
$$

But then $\lambda$ is inaccessible, and since $C_{n} \cap \lambda$ is unbounded, $\lambda \in I_{n}$.

(4) $\Rightarrow(3)$ : Suppose that $R$ is a $\Delta_{n+1}$ subset of $V_{\kappa}$ and $\Phi$ is a $\Pi_{1}^{1}$ sentence such that

$$
\left\langle V_{\kappa}, \in, R\right\rangle \models \Phi .
$$

Let $\varphi\left(x, y_{0}, \ldots, y_{k}\right)$ be a $\Sigma_{n+1}$ formula and $\psi\left(x, z_{0}, \ldots, z_{l}\right)$ a $\Pi_{n+1}$ formula that define $R$ in $V_{\kappa}$ with parameters $a_{0}, \ldots, a_{k}$ and $b_{0}, \ldots, b_{l}$, respectively. 
Thus,

$$
\left\langle V_{\kappa}, \in, R\right\rangle \models \forall x\left(R x \leftrightarrow \varphi\left(x, a_{0}, \ldots, a_{k}\right) \leftrightarrow \psi\left(x, b_{0}, \ldots, b_{l}\right)\right) .
$$

Let $\Phi^{\prime}\left(y_{0}, \ldots, y_{k}\right)$ be the $\Pi_{1}^{1}$ formula (with $y_{0}, \ldots, y_{k}$ as the only free individual variables) obtained by substituting every occurrence of the formula $R x$ in $\Phi$ by the formula $\varphi\left(x, y_{0}, \ldots, y_{k}\right)$. Then, clearly, $V_{\kappa} \models \Phi^{\prime}\left(a_{0}, \ldots, a_{k}\right)$.

Hence, there is $\lambda \in I_{n}$ such that

$$
V_{\lambda} \models \Phi^{\prime}\left(a_{0}, \ldots, a_{k}\right) \wedge \forall x\left(\varphi\left(x, a_{0}, \ldots, a_{k}\right) \leftrightarrow \psi\left(x, b_{0}, \ldots, b_{l}\right)\right) .
$$

But since $V_{\lambda} \preccurlyeq_{n} V_{\kappa}, R \cap V_{\lambda}=\left\{x: V_{\lambda} \models \varphi\left(x, a_{0}, \ldots, a_{k}\right)\right\}$. Therefore,

$$
\left\langle V_{\lambda}, \in, R \cap V_{\lambda}\right\rangle \models \Phi \text {. }
$$

Notice that in the proof of $(4) \Rightarrow(3)$ above, we have not made use of the fact that $\lambda$ was inaccessible. Thus an inaccessible cardinal $\kappa$ is $\Sigma_{n}$-w.c. iff $\kappa$ reflects $\Pi_{1}^{1}$ sentences (in the language with $\in$ only) to some $\lambda<\kappa$ such that $V_{\lambda} \preccurlyeq n V_{\kappa}$.

Leshem [9] has proved that if $\kappa$ is Mahlo, then the set of $\Sigma_{\omega}$-w.c. cardinals below $\kappa$ is stationary. So, all these cardinals are, consistency-wise, below a Mahlo cardinal.

Let us recall from [2] that a subset $C$ of a cardinal $\kappa$ is a $\prod_{n}-c l u b$ iff $C$ is a club subset of $\kappa$ that is definable over $V_{\kappa}$ by means of a $\widetilde{\Pi}_{n}$ formula,

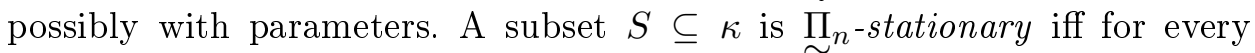

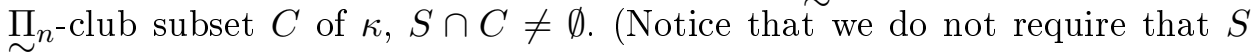
itself be $\Pi_{n}$-definable.) Finally, $\kappa$ is a $\prod_{n}$-Mahlo cardinal iff it is inaccessible and the set of all inaccessible cardinals below $\kappa$ is $\Pi_{n}$-stationary. For more information about $\prod_{\sim}$-Mahlo cardinals see [2] and [4]. The next fact shows that $\Sigma_{n}$-w.c. cardinals are $\underset{\sim}{\prod_{n}}$-Mahlo, and that the least $\underset{\sim}{\prod_{n}}$-Mahlo cardinal is not $\Sigma_{n}$-w.c.

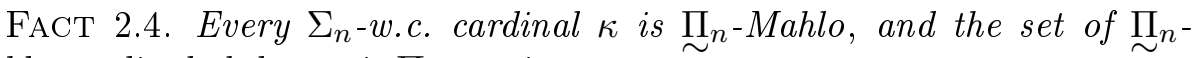
Mahlo cardinals below $\kappa$ is $\prod_{n}$-stationary.

Proof. Suppose that $\kappa$ is $\Sigma_{n^{-}}$w.c. Let $C$ be a $\underset{\sim}{\prod^{-}}$-club of $\kappa$, i.e., $C$ is a club on $\kappa$ which is definable over $V_{\kappa}$ by means of a $\Pi_{n}$ formula with parameters. Let $\Phi$ the $\Pi_{1}^{1}$ sentence expressing that $\kappa$ is inaccessible. Let $\varrho$ be the first-order sentence expressing that $C$ is unbounded. Then

$$
\left\langle V_{\kappa}, \in, C\right\rangle \models \Phi \wedge \varrho .
$$

So, there is $\alpha<\kappa$ such that

$$
\left\langle V_{\alpha}, \in, C \cap V_{\alpha}\right\rangle \models \Phi \wedge \varrho .
$$

Therefore $\alpha$ is inaccessible, and since $C \cap V_{\alpha}=C \cap \alpha$ is unbounded in $\alpha$, $\alpha \in C$. 
Note that "every $\prod_{\sim}$-club of $\kappa$ contains an inaccessible cardinal" is expressible by a first-order sentence. Therefore, the above argument shows that there is a $\prod_{n}$-stationary set of $\underset{\sim}{\prod_{n}}$-Mahlo cardinals below $\kappa$.

Recall $\kappa$ is a $\Sigma_{\omega}$-Mahlo cardinal iff it is $\prod_{n}$-Mahlo for every $n \in \omega$. In [4] it is shown that every $\Sigma_{\omega}$-w.c. cardinal is $\Sigma_{\omega}$-Mahlo, and that the set of $\Sigma_{\omega}$-Mahlo cardinals below a $\Sigma_{\omega}$-w.c. cardinal is $\Sigma_{\omega}$-stationary. However, also from [4], if $\kappa$ is $\Pi_{n+1}$-Mahlo, then the set of $\Sigma_{n}$-w.c. cardinals below $\kappa$ is $\Pi_{n+1}$-stationary.

\subsubsection{The tree property}

Definition 2.5. Let $\kappa$ be a cardinal and $n \in \omega$. A tree $T=\left\langle T, \leq_{T}\right\rangle$ with $T \subseteq V_{\kappa}$ is a $\Sigma_{n}$-tree (over $V_{\kappa}$ ) iff there are $\Sigma_{n}$ formulas $\varphi_{T}(x), \varphi_{\leq_{T}}(x, y)$ and $\varphi_{\mathrm{ht}_{T}}(x, y)$, possibly with parameters in $V_{\kappa}$, such that for every $t, t^{\prime} \in V_{\kappa}$ and every $\alpha<\kappa$,

$$
\begin{array}{cll}
t \in T \quad \text { iff } & V_{\kappa}=\varphi_{T}(t), \\
t \leq_{T} t^{\prime} \quad \text { iff } & V_{\kappa}=\varphi_{\leq_{T}}\left(t, t^{\prime}\right), \\
t \in T_{\alpha} & \text { iff } & V_{\kappa}=\varphi_{\mathrm{ht}_{T}}(t, \alpha),
\end{array}
$$

where $T_{\alpha}$ denotes the $\alpha$ th level of the tree $T$. Similarly, we define the notion of $\Pi_{n}$-tree by substituting $\Pi_{n}$ for $\Sigma_{n}$ in the above definition. Moreover, $T$ is a $\Delta_{n}$-tree iff $T$ is both a $\Sigma_{n}$-tree and a $\Pi_{n}$-tree. Finally, $T$ is a $\Sigma_{\omega}$-tree iff $T$ is a $\Sigma_{n}$-tree for some $n \in \omega$.

Definition 2.6. Let $\kappa$ be a cardinal and $n \in \omega . \kappa$ has the $\Sigma_{n}$-tree property iff $\kappa$ is inaccessible and every $\kappa$-tree which is a $\Sigma_{n}$-tree has a cofinal branch. The $\Pi_{n}$-tree property, $\Delta_{n}$-tree property, and $\Sigma_{\omega}$-tree property are defined analogously.

Lemma 2.7. For every $n \in \omega$, if $\kappa$ is $\Sigma_{n}$-w.c., then $\kappa$ has the $\Sigma_{n}$-tree property.

Proof. Suppose that $\kappa$ is a $\Sigma_{n}$-w.c. cardinal and let $T$ be a $\kappa$-tree which is a $\Sigma_{n}$-tree over $V_{\kappa}$. Suppose that $T$ does not have a branch of length $\kappa$. So, since $\kappa$ is regular, every branch of $T$ belongs to $V_{\kappa}$.

Let $\Phi$ be the $\Pi_{1}^{1}$ sentence expressing that $\kappa$ is inaccessible.

Let $\Psi$ be the following $\Pi_{1}^{1}$ sentence:

$$
\forall B(B \text { is a branch of } T \rightarrow \exists x B=x) .
$$

Let $F$ be the function with domain $\kappa$ such that $F(\alpha)=T_{\alpha}$, the $\alpha$ th level of $T$. Since $t \in T_{\alpha}$ is a $\Sigma_{n}$ fact over $V_{\kappa}, F$ is $\Delta_{n+1}$-definable over $V_{\kappa}$. Let $\varphi$ be the following first-order sentence:

$$
\forall \alpha(\alpha \text { is an ordinal } \rightarrow \exists x F(\alpha)=x) .
$$


Thus,

$$
\left\langle V_{\kappa}, \in, T, F\right\rangle \models \Phi \wedge \Psi \wedge \varphi .
$$

Hence, there is $\lambda<\kappa$ such that

$$
\left\langle V_{\lambda}, \in, T \cap V_{\lambda}, F \cap V_{\lambda}\right\rangle \models \Phi \wedge \Psi \wedge \varphi .
$$

Fix some $t \in T_{\lambda}$. Let $\operatorname{pred}(t)=\left\{t^{\prime} \in T: t^{\prime}<_{T} t\right\}$. It is clear that $\operatorname{pred}(t)$ is a branch through $T \cap V_{\lambda}$. So, $\operatorname{pred}(t) \in V_{\lambda}$, and hence, since $\lambda$ is inaccessible, $|\operatorname{pred}(t)|<\lambda$. A contradiction.

Corollary 2.8. If $\kappa$ is $\Sigma_{\omega}$-w.c., then $\kappa$ has the $\Sigma_{\omega}$-tree property.

2.1.2. The partition property. Recall that if $\kappa$ is a cardinal and $n>0$ is a natural number, $[\kappa]^{n}$ is the set of all subsets of $\kappa$ with exactly $n$ elements.

Given a cardinal $\kappa$, natural numbers $n, m(n>0)$, and a function $f$ : $[\kappa]^{n} \rightarrow m$, a set $H \subseteq \kappa$ is said to be $f$-homogeneous iff $f^{\prime \prime}[H]^{n}=\{i\}$ for some $i \in m$.

DeFinition 2.9. Let $\kappa$ be a cardinal. Then $\kappa$ has the $\Sigma_{n}$-partition property iff $\kappa$ is an inaccessible cardinal and for every function $f:[\kappa]^{2} \rightarrow\{0,1\}$ that is $\Sigma_{n}$-definable over $V_{\kappa}$ there exists an $f$-homogeneous set of cardinality $\kappa$. We write $\kappa \stackrel{\Sigma_{n}}{\longrightarrow}(\kappa)^{2}$ to indicate that $\kappa$ has the $\Sigma_{n}$-partition property. The $\Sigma_{\omega}$-partition property is defined analogously, and we write $\kappa \stackrel{\Sigma_{\omega}}{\longrightarrow}(\kappa)^{2}$.

Lemma 2.10. For every $n \in \omega, n>0$, if $\kappa$ has the $\Sigma_{n}$-tree property, then $\kappa \stackrel{\Sigma_{n}}{\longrightarrow}(\kappa)^{2}$.

Proof. Let $F:[\kappa]^{2} \rightarrow\{0,1\}$ be $\Sigma_{n}$-definable over $V_{\kappa}$. Let $\varphi(x, y, z)$ be a $\Sigma_{n}$ formula, possibly with parameters in $V_{\kappa}$, that defines it.

For every $\beta<\kappa$, let $f_{\beta}: \beta \rightarrow\{0,1\}$ be such that for all $\alpha<\beta, f_{\beta}(\alpha)=$ $F(\{\alpha, \beta\})$. Let $T=\left\{f_{\beta} \mid \gamma: \gamma \leq \beta<\kappa\right\}$ be ordered by extension. Note that $T$ is $\Sigma_{n}$-definable over $V_{\kappa}$ :

$t \in T$ iff $V_{\kappa}=\exists \beta, \gamma(\gamma \leq \beta \wedge \operatorname{dom}(t)=\gamma \wedge(\forall \alpha<\gamma)(\exists i \in\{0,1\})(\varphi(\alpha, \beta, i)))$.

It is clear that for every $\beta<\kappa$, we have: $t \in T_{\beta}$ iff $t \in T$ and $\operatorname{dom}(t)=\beta$. So, $T$ is a $\Sigma_{n}$-tree. Moreover, ht $(T)=\kappa$, and since for every $\beta<\kappa, T_{\beta} \subseteq 2^{\beta}$, and $\kappa$ is inaccessible, $\left|T_{\beta}\right|<\kappa$. Therefore $T$ is a $\kappa$-tree.

Since $\kappa$ has the $\Sigma_{n}$-tree property, there is a cofinal branch $B$ through $T$. Let $\left\{t_{\xi}: \xi<\kappa\right\}$ be an increasing enumeration of $B$ so that $\operatorname{dom}\left(t_{\xi}\right)=\xi$ for all $\xi<\kappa$. For every $i \in\{0,1\}$, let

$$
H_{i}=\left\{\xi<\kappa: t_{\xi}\langle\xi, i\rangle \in B\right\}
$$

We claim that for every $i \in\{0,1\}, H_{i}$ is a homogeneous subset of $\kappa$ for $F$. Fix $\alpha, \beta, \gamma \in H_{i}$ with $\alpha<\beta<\gamma$. Since $t_{\alpha}\langle\alpha, i\rangle \subseteq t_{\beta}$ and $t_{\beta}\langle\beta, i\rangle \subseteq t_{\gamma}$,

$$
F(\{\alpha, \beta\})=t_{\beta}(\alpha)=i=t_{\gamma}(\beta)=F(\{\beta, \gamma\}) .
$$


So, the $H_{i}$ are homogeneous for $i \in\{0,1\}$. Since $|B|=\kappa$, either $\left|H_{0}\right|=\kappa$ or $\left|H_{1}\right|=\kappa$. Therefore, $\kappa \stackrel{\Sigma_{n}}{\longrightarrow}(\kappa)^{2}$.

Corollary 2.11. If $\kappa$ has the $\Sigma_{\omega}$-tree property, then $\kappa \stackrel{\Sigma_{\omega}}{\longrightarrow}(\kappa)^{2}$.

Lemma 2.12 (E. Kranakis, [8]). Assume $V=L$. For every $n>0, \kappa \stackrel{\Sigma_{n}}{\longrightarrow}$ $(\kappa)^{2}$ implies that for every $\Pi_{1}^{1}$ formula $\Phi\left(x_{0}, \ldots, x_{k}\right)$ and $a_{0}, \ldots, a_{k} \in L_{\kappa}$ such that $L_{\kappa} \models \Phi\left(a_{0}, \ldots, a_{k}\right)$, there is $\lambda<\kappa$ with $L_{\lambda} \preccurlyeq_{n} L_{\kappa}$ such that $L_{\kappa} \models \Phi\left(a_{0}, \ldots, a_{k}\right)$.

Finally, we have:

Theorem 2.13. $(V=L)$ Let $\kappa$ be a cardinal. Then for every $n \geq 1$ the following are equivalent:

(1) $\kappa$ is a $\Sigma_{n}$-w.c. cardinal.

(2) $\kappa$ has the $\Sigma_{n}$-tree property.

(3) $\kappa \stackrel{\Sigma_{n}}{\longrightarrow}(\kappa)^{2}$.

Proof. (1) $\Rightarrow$ (2) follows from Lemma 2.7 .

$(2) \Rightarrow(3)$ follows from Lemma 2.10 .

Since $L=\kappa \stackrel{\Sigma_{n}}{\longrightarrow}(\kappa)^{2}$, by definition, $\kappa$ is inaccessible in $L$. The rest of implication $(3) \Rightarrow(1)$ follows from Lemma 2.12 (this is the only place where $V=L$ is used) and Proposition 2.3.

Corollary 2.14. $(V=L)$ Let $\kappa$ be a cardinal. Then the following are equivalent:

(1) $\kappa$ is $\Sigma_{\omega}$-w.c.

(2) $\kappa$ has the $\Sigma_{\omega}$-tree property.

(3) $\kappa \stackrel{\Sigma_{\omega}}{\longrightarrow}(\kappa)^{2}$.

2.2. Generic absoluteness for projective ccc posets

Definition 2.15. $L(\mathbb{R})^{M}$ is a $\Sigma_{n}$-w.c. (resp. $\Sigma_{\omega}$-w.c.) Solovay model over $V \subseteq M$ iff $M$ satisfies:

(1) For every $x \in \mathbb{R}, \omega_{1}$ is a $\Sigma_{n}$-w.c. (resp. $\Sigma_{\omega}$-w.c.) cardinal in $V[x]$.

(2) Every $x \in \mathbb{R}$ is small-generic over $V$.

Notice that since every $\Sigma_{n}$-w.c. (resp. $\Sigma_{\omega}$-w.c.) cardinal is inaccessible, Lemma 1.1 also holds for $\Sigma_{n}$-w.c. (resp. $\Sigma_{\omega}$-w.c.) Solovay models.

We will make use of the following property of $\Sigma_{n}$-w.c. cardinals:

Lemma 2.16. Let $n \geq 1$. Suppose that $\kappa$ is a $\Sigma_{n}$-w.c. cardinal and $\mathbb{P}$ is a $\kappa$-cc poset that is $\Sigma_{n}$-definable (with parameters) over $V_{\kappa}$. If $X \subseteq \mathbb{P}$ has cardinality less than $\kappa$, then there is a complete subposet $\mathbb{Q}$ of $\mathbb{P}$, also of cardinality less than $\kappa$, such that $X \subseteq \mathbb{Q}$. 
Proof. Let $X \subseteq \mathbb{P}$ with $|X|<\kappa$. Since $\kappa$ is inaccessible, there is a cardinal $\lambda<\kappa$ with $X \subseteq V_{\lambda}$.

Let $R=\{D: D$ is a maximal antichain of $\mathbb{P}\}$. Since $\mathbb{P}$ is $\kappa$-cc, $R \subseteq V_{\kappa}$. For all $D \in V_{\kappa}, D \in R$ iff $V_{\kappa}$ satisfies:

$$
D \subseteq \mathbb{P} \wedge \forall x, y \in D\left(x \neq y \rightarrow x \perp_{\mathbb{P}} y\right) \wedge \forall z\left(z \in \mathbb{P} \rightarrow \exists y \in D\left(\neg z \perp_{\mathbb{P}} y\right)\right) .
$$

Note that the formula above is the conjunction of a $\Sigma_{n}$ formula and a $\Pi_{n}$ formula. Hence, $R$ is a $\Delta_{n+1}$ predicate in $V_{\kappa}$.

Let $\Phi$ be the conjunction of the following sentences of the second-order language of type $\left\{\in, \mathbb{P}, \leq_{\mathbb{P}}, \perp_{\mathbb{P}}, R\right\}$ :

(1) $\leq_{\mathbb{P}}$ is a partial order with field $\left(\leq_{\mathbb{P}}\right)=\mathbb{P}$.

(2) $\perp_{\mathbb{P}}$ is the incompatibility relation of $\left\langle\mathbb{P}, \leq_{\mathbb{P}}\right\rangle$.

(3) $\forall Y\left(Y \subseteq \mathbb{P} \wedge \forall x y\left(Y x \wedge Y y \wedge x \neq y \rightarrow x \perp_{\mathbb{P}} y\right)\right.$

$\left.\wedge \forall z\left(\mathbb{P} z \rightarrow \exists y\left(Y y \wedge \neg y \perp_{\mathbb{P}} z\right)\right) \rightarrow \exists x(R x \wedge Y=x)\right)$, i.e, every maximal antichain of $\mathbb{P}$ belongs to $R$.

Notice that (1) and (2) are first-order, and (3) is $\Pi_{1}^{1}$.

We have

$$
\left\langle V_{\kappa}, \in, \mathbb{P}, \leq_{\mathbb{P}}, \perp_{\mathbb{P}}, R\right\rangle \models \Phi .
$$

So, since $\kappa$ is $\Sigma_{n}$-w.c., there is $\alpha<\kappa$ with $\lambda<\alpha$ such that

$$
\left\langle V_{\alpha}, \in, \mathbb{P} \cap V_{\alpha}, \leq_{\mathbb{P}} \cap V_{\alpha}, \perp_{\mathbb{P}} \cap V_{\alpha}, R \cap V_{\alpha}\right\rangle \models \Phi .
$$

Let $\mathbb{Q}=\left\langle\mathbb{P} \cap V_{\alpha}, \leq_{\mathbb{P}} \cap V_{\alpha}, \perp_{\mathbb{P}} \cap V_{\alpha}\right\rangle$. So, $|\mathbb{Q}|<\kappa$. By (1) and (2), $\mathbb{Q}$ is a subposet of $\mathbb{P}$ that preserves the incompatibility relation of $\mathbb{P}$. Since $\lambda<\alpha$, we have $X \subseteq \mathbb{P} \cap V_{\alpha}$. Finally, let $D$ be a maximal antichain of $\mathbb{Q}$. Then, by (3), $D \in R \cap V_{\alpha}$. So since $D \in R$, it follows that $D$ is a maximal antichain of $\mathbb{P}$. This shows that $\mathbb{Q}$ is a complete subposet of $\mathbb{P}$ of cardinality less than $\kappa$ which includes $X$.

For $\alpha$ an ordinal, we shall write $\operatorname{Coll}_{\alpha}$ for the Levy collapse below $\alpha$, instead of the usual and more cumbersome $\operatorname{Coll}(\omega,<\alpha)$.

Theorem 2.17. Let $n \geq 1$. Suppose $L(\mathbb{R})^{M}$ is a $\Sigma_{n}$-w.c. Solovay model over $V$ and $\mathbb{P}$ is a ccc poset which is, in $M, \Sigma_{n}$-definable (with parameters) over $H\left(\omega_{1}\right)$. Then the $L(\mathbb{R})$ of any $\mathbb{P}$-extension of $M$ is also a $\Sigma_{n}$-w.c. Solovay model over $V$.

Proof. Let $\kappa=\omega_{1}^{M}$. Force over $M$ with Woodin's partial ordering $\mathbb{W}$ (see Lemma 1.1) to obtain a Coll $\kappa^{\text {-generic }} C$ over $V$ so that $\mathbb{R}^{M}=\mathbb{R}^{V[C]}$. Notice that for a generic filter $G \subseteq \mathbb{P}, G$ is $\mathbb{P}$-generic over $M$ iff it is $\mathbb{P}$-generic over $V[C]$ and, moreover, $\mathbb{R}^{M[G]}=\mathbb{R}^{V[C][G]}$. Thus, to prove the theorem it will be enough to show that every real in $V[C][G]$ is generic over $V$ for some forcing notion $\mathbb{P}$ in $V$ that is countable in $V[C][G]$. 
Let $\dot{\mathbb{P}}$ be a Coll $_{\kappa}$-name for $\mathbb{P}$ in $V$. By the Factor Lemma for the Levy collapse, we may assume that the parameters of the definition of $\mathbb{P}$ are in $V$. Further, since the Levy collapse is homogeneous, we may assume that $\Vdash_{\mathrm{Coll}_{\kappa}}$ " $\dot{P}$ is a poset". Notice that Coll $_{\kappa}$ is definable by means of a $\Sigma_{1}$ and a $\Pi_{1}$ formula without parameters over $V_{\kappa}$ (see [2]). Hence, for $n \geq 1, \operatorname{Coll}_{\kappa} * \dot{\mathbb{P}}$ is a poset which is $\Sigma_{n}$-definable over $V_{\kappa}$, possibly with parameters.

Let $x$ be a real in $V[C][G]$. Let $\dot{x}$ be a simple $\operatorname{Coll}_{\kappa} * \dot{\mathbb{P}}$-name for $x$ in $V$, and let $X$ be the set of all conditions of $\operatorname{Coll}_{\kappa} * \dot{\mathbb{P}}$ in $T C(\dot{x})$. Since $\operatorname{Coll}_{\kappa} * \dot{\mathbb{P}}$ is $\kappa$-cc, $|X|<\kappa$. So, by Lemma 2.16 , there is a complete subposet $\mathbb{Q}$ of $\operatorname{Coll}_{\kappa} * \dot{\mathbb{P}}$ such that $X \subseteq \mathbb{Q}$ and $\mathbb{Q}$ has cardinality less than $\kappa$. Let $H=(C * G) \cap \mathbb{Q}$. Then $H$ is $\mathbb{Q}$-generic over $V$ and $\dot{x}[H]=\dot{x}[C * G]=x$. This completes the proof of the theorem since it shows that $x$ is generic over $V$ for the countable poset $\mathbb{Q}$.

Corollary 2.18 .

(1) For every $n \geq 1$, Con(ZFC + there exists a $\Sigma_{n}$-w.c. cardinal) implies $\mathrm{Con}\left(\mathrm{ZFC}+\bar{L}(\mathbb{R})\right.$-absoluteness for $\sum_{n+1}^{1}$ ccc posets $)$.

(2) Con $\left(\mathrm{ZFC}+\right.$ there exists a $\Sigma_{\omega}$-w.c. cardinal $)$ implies $\operatorname{Con}(\mathrm{ZFC}+L(\mathbb{R})$ absoluteness for projective ccc posets).

Proof. (1): Suppose $\kappa$ is $\Sigma_{n}$-w.c. Force with $\operatorname{Coll}_{\kappa}$ so that the $L(\mathbb{R})$ of the generic extension $M$ is a $\Sigma_{n}$-w.c. Solovay model over $V$. By Theorem 2.17 and Lemma 1.3, $L(\mathbb{R})$-absoluteness holds in $M$ for ccc posets that are $\Sigma_{n}$ definable, with parameters, in $H\left(\omega_{1}\right)$, and hence, for $\sum_{n+1}^{1}$ ccc posets.

Recall that for $\Gamma$ a class of posets, a poset $\mathbb{P}$ is $\Gamma$-productive-ccc iff it is ccc and for every ccc poset $\mathbb{Q}$ in $\Gamma, \mathbb{P} \times \mathbb{Q}$ is ccc.

Let $\Gamma_{n}$ be the class of all $\sum_{n+1}^{1}$ ccc posets, and let $\Gamma_{\omega}$ be the class of all projective ccc forcing notions. Then, as in [2], we can show:

THEOREM 2.19 .

(1) If $L(\mathbb{R})^{M}$ is a $\Sigma_{n}$-w.c. Solovay model, then in $L(\mathbb{R})^{M}$ every ccc poset is $\Gamma_{n}$-productive-ccc.

(2) If $L(\mathbb{R})^{M}$ is a $\Sigma_{\omega}$-w.c. Solovay model, then in $L(\mathbb{R})^{M}$ every ccc poset is $\Gamma_{\omega}$-productive-ccc.

Proof. (1): Suppose $L(\mathbb{R})^{M}$ is a $\Sigma_{n}$-w.c. Solovay model over $V$, and in $L(\mathbb{R})^{M}, \mathbb{P}$ is a ccc poset and $\mathbb{Q}$ is a poset in the class $\Gamma_{n}$.

It is known (see [7]) that there is a ccc poset $\mathbb{Q}^{*}$ in $\Gamma_{n}$ such that $\mathbb{Q}$ completely embeds into $\mathbb{Q}^{*}$, and if $G$ is $\mathbb{Q}^{*}$-generic over some model $M$, then $M[G]$ is of the form $M[g]$ for some real $g$.

Let $\mathbb{Q}^{*}$ be as above, and suppose $\tau$ is a $\mathbb{Q}^{*}$-name for an uncountable antichain of $\mathbb{P}, \tau \in L(\mathbb{R})^{M}$. Let $\varphi_{\mathbb{P}}(x), \varphi_{\leq \mathbb{P}}(x, y)$ and $\varphi_{\perp_{\mathbb{P}}}(x, y)$ be formulas with only reals and ordinals as parameters that define, respectively, $\mathbb{P}$, 
$\leq_{\mathbb{P}}$, and $\perp_{\mathbb{P}}$ in $L(\mathbb{R})^{M}$, and let $\varphi_{\mathbb{Q}^{*}}(x), \varphi_{\leq_{\mathbb{Q}^{*}}}(x, y)$, and $\varphi_{\perp_{\mathbb{Q}^{*}}}(x, y)$ be $\Sigma_{n+1}^{1}$ formulas with real parameters that define, respectively, $\mathbb{Q}^{*}, \leq \mathbb{Q}^{*}$, and $\perp_{\mathbb{Q}^{*}}$. Thus, there is a formula $\varphi(x, y)$ with only reals and ordinals as parameters such that the following holds in $L(\mathbb{R})^{M}$ :

(i) For all $p, a$, if $\varphi(p, a)$, then $\varphi_{\mathbb{Q}^{*}}(p)$ and $\varphi_{\mathbb{P}}(a)$.

(ii) For all $p, q, a, b$, if $\varphi(p, a), \varphi(q, b)$, and not $\varphi_{\perp_{\mathbb{Q}^{*}}}(p, q)$, then $\varphi_{\perp_{\mathbb{P}}}(a, b)$.

(iii) For all $p, a, \varphi(p, a)$ iff $\langle p, \check{a}\rangle \in \tau$.

Suppose $G$ is $\mathbb{Q}^{*}$-generic over $L(\mathbb{R})^{M}$. So, $G$ is also generic over $M$. Let $N$ be the $L(\mathbb{R})$ of $L(\mathbb{R})^{M}[G]$. Clearly, since $M[G]$ and $L(\mathbb{R})^{M}[G]$ have the same reals, $N=L(\mathbb{R})^{M[G]}$. Thus, by Lemma 1.3 and Theorem 2.17, (i) and (ii) above hold in $N$. Since $G$ is easily coded by a real, $G \in N$. In $N$, let $A=\{a: \exists p \in G \varphi(p, a)\}$. Notice that, by (iii) above, $\tau[G] \subseteq A$, and so $A$ is an uncountable set in $N$. Also, for every $a \in A, N \models \varphi_{\mathbb{P}}(a)$. Let $\mathbb{P}^{N}$ and $\leq_{\mathbb{P}}^{N}$ be the sets defined in $N$ by the formulas $\varphi_{\mathbb{P}}(x)$ and $\varphi_{\leq_{\mathbb{P}}}(x, y)$, respectively. Then $N \models "\left\langle\mathbb{P}^{N}, \leq \mathbb{P}^{N}\right\rangle$ is a ccc poset". So, since

$$
N \models \text { " } A \text { is an uncountable subset of } \mathbb{P}^{N "} \text {, }
$$

we have

$$
N \models " \exists p, q, a, b\left(\varphi(p, a) \wedge \varphi(q, b) \wedge \neg \varphi_{\perp_{\mathbb{Q}^{*}}}(p, q) \wedge \neg \varphi_{\perp_{\mathbb{P}}}(a, b)\right) " .
$$

Therefore, by 1.3 and 2.17 ,

$$
L(\mathbb{R})^{M} \models " \exists p, q, a, b\left(\varphi(p, a) \wedge \varphi(q, b) \wedge \neg \varphi_{\perp_{\mathbb{Q}^{*}}}(p, q) \wedge \neg \varphi_{\perp_{\mathbb{P}}}(a, b)\right) ",
$$

which contradicts (ii) above.

Now suppose $H$ is $\mathbb{Q}$-generic over $L(\mathbb{R})^{M}$. Let $G$ be $\mathbb{Q}^{*}$-generic over $L(\mathbb{R})^{M}$ such that

$$
L(\mathbb{R})^{M}[H] \subseteq L(\mathbb{R})^{M}[G] .
$$

Since $\mathbb{P}$ is ccc in $L(\mathbb{R})^{M}[G]$, it is also ccc in $L(\mathbb{R})^{M}[H]$.

Corollary 2.20. If $L(\mathbb{R})^{M}$ is a $\Sigma_{n}$-w.c. Solovay model over $V$, then in $M$ there are no ${\underset{\sim n+1}{1}}_{n+1}$ Suslin trees. And if $L(\mathbb{R})^{M}$ is a $\Sigma_{\omega}$-w.c. Solovay model over $V$, then in $M$ there are no projective Suslin trees.

Proof. If $T$ is a $\sum_{n+1}^{1}$ Suslin tree, then $T \times T$ with the product ordering is a $\underset{\sim}{\sum_{n+1}}$ poset which is not ccc (see [6]).

3. The strength of generic absoluteness under projective ccc forcing notions. In this section we shall prove the following:

THEOREM 3.1. If $\Sigma_{4}^{1}$-absoluteness holds for $\Delta_{3}^{1}$ ccc forcing notions, then $\omega_{1}$ is a $\Sigma_{1}$-w.c. cardinal in $L$.

Proof. Suppose towards a contradiction that $\omega_{1}$ is not $\Sigma_{1}$-w.c. in $L$. We know (see [2]) that $\omega_{1}$ is inaccessible in $L$ and, in fact, $\omega_{1}$ is inaccessible to 
reals, i.e., $\omega_{1}^{L[x]}$ is countable for every real $x$. Hence, by Theorem 2.13, there is, in $L$, an Aronszajn tree $T=\left\langle T, \leq_{T}\right\rangle$ whose nodes are elements of $2^{<\omega_{1}}$ and which is a $\Sigma_{1}$-tree over $L_{\omega_{1}}$.

We need the following version of the Silver tree $S_{T}$ for $T$ (See [5]): For every set $M$ and every $X \subseteq M$, let $H^{M}(X)$ denote the Skolem hull of $X$ in $M$. Then the Silver tree $S_{T}$ for $T$ is defined as follows:

(1) $\langle\alpha, \beta, a\rangle \in S_{T}$ iff

(a) $\alpha<\beta<\omega_{1}$,

(b) $a \in L_{\beta}$ is a function with $\alpha \subseteq \operatorname{dom}(a)$,

(c) $L_{\beta}=H^{L_{\beta}}(\alpha \cup\{a\})$,

(d) $a\lceil\alpha \in T$.

(2) $\langle\alpha, \beta, a\rangle \leq_{S_{T}}\langle\gamma, \delta, c\rangle$ iff

(a) $\alpha \leq \gamma$,

(b) $L_{\beta}=\mu$ " $H^{L_{\delta}}(\alpha \cup\{c\})$, where $\mu$ is Mostowski's transitive collapse function, and $\mu(c)=a$.

Note that if $\langle\alpha, \beta, a\rangle \in S_{T}$, then $\langle\alpha, \beta, a\rangle$ is a node of height $\alpha$.

Lemma 3.2 (J. H. Silver, see [5]). $S_{T}$ is an Aronszajn tree in $L$ such that in any model of ZFC (extending $L$ ), if there is a branch of length $\omega_{1}$ through $S_{T}$, then $\operatorname{cf}\left(\omega_{1}\right)=\omega$.

An important fact for our purposes is that the complexity of $S_{T}$ is the same as that of $T$. That is:

Lemma 3.3. For all $n \geq 1$, if $T \subseteq 2^{<\omega_{1}}$ is a $\Sigma_{n}$-tree (resp. $\Pi_{n}$-tree) over $L_{\omega_{1}}$, then $S_{T}$ is also a $\Sigma_{n}$-tree (resp. $\Pi_{n}$-tree) over $L_{\omega_{1}}$.

Proof. Fix some recursive enumeration $\left\langle\varphi_{i}: i \in \omega\right\rangle$ of all formulas of the language of set theory of the form $\exists \bar{x} \varphi(\bar{y}, \bar{z}, \bar{x})$, where $\bar{y}, \bar{z}, \bar{x}$ are finite sequences of variables and $\bar{x}$ is non-empty. We use the following notational conventions: given a formula $\varphi_{i}$, we denote by $\varphi_{i}^{\prime}$ the formula resulting from the removal of the first block of existential quantifiers of $\varphi_{i}$. Also, $\exists \bar{y} \varphi_{i}$ denotes the formula resulting by adding the block of existential quantifiers $\exists \bar{y}$ to the formula $\varphi_{i}$. Note that the maps $\varphi_{i} \mapsto \varphi_{i}^{\prime}$ and $\varphi_{i} \mapsto \exists \bar{y} \varphi_{i}$ are recursive.

If $x$ is an ordered pair, then let $(x)_{0}$ and $(x)_{1}$ denote, respectively, the first and second coordinates of $x$.

For every set $M \in L$, we define the function $r^{M}$ from $\omega \times M^{<\omega}$ to $M^{<\omega} \times M^{<\omega}$ as follows: for all $i \in \omega$ and every $b \in M^{<\omega}$,

$$
r^{M}(i, b)= \begin{cases}\text { the }<{ }_{L} \text {-least } a \in M^{<\omega} \times M^{<\omega} \text { such that } \\ M \models \varphi_{i}^{\prime}\left((a)_{0}, b,(a)_{1}\right) & \text { if } M \models \exists y \varphi_{i}(b), \\ \langle\emptyset, \emptyset\rangle & \text { if } M \not \models \exists y \varphi_{i}(b) .\end{cases}
$$


Let $\mathrm{Sk}^{M}$ be the function from $\omega \times M^{<\omega}$ into $M^{<\omega}$ defined by $\mathrm{Sk}^{M}(i, b)=$ $\left(r^{M}(i, b)\right)_{0}$ for every $i \in \omega$ and $b \in M^{<\omega}$.

Claim 3.4. $(V=L)$ For every set $M$, the functions $r^{M}$ and $\mathrm{Sk}^{M}$ are $\Delta_{1}$ with $M$ as a parameter.

Proof. We only need to show that $r^{M}$ is $\Delta_{1}$. Let $\operatorname{Sat}(x, y, z)$ denote the satisfaction relation for sets, i.e., $\operatorname{Sat}(x, y, z)$ iff the set $x$ satisfies the formula $y$ with the sequence $z$ of elements of $x$. Notice that this is a $\Delta_{1}$ relation.

For every $i \in \omega$, and every $b \in M^{<\omega}, r^{M}(i, b)=a$ iff

(1) $a$ is an ordered pair, and $(a)_{0},(a)_{1} \in M^{<\omega}$.

(2) Either $\operatorname{Sat}\left(M, \exists y \varphi_{i}, b\right)$ and

(a) $\operatorname{Sat}\left(M, \varphi_{i}^{\prime},(a)_{0}^{-} b-(a)_{1}\right)$,

(b) $(\forall c, d \in M)\left(\operatorname{Sat}\left(M, \varphi_{i}^{\prime}, c \frown b \frown d\right) \rightarrow a<_{L}\langle c, d\rangle\right)$,

(3) or $\neg \operatorname{Sat}\left(M, \exists \bar{y} \varphi_{i}, b\right)$ and $(\forall c, d \in M)\left(a \leq_{L}\langle c, d\rangle\right)$.

Since $<_{L}$ is a $\Delta_{1}$ relation, (1), (2), and (3) can be written as both $\Sigma_{n}$ and $\Pi_{n}$ sentences. Hence, $r^{M}$ is a $\Delta_{1}$ function.

Therefore, the functions $M \mapsto r^{M}$ and $M \mapsto \mathrm{Sk}^{M}$ are $\Delta_{1}$ definable in $L$ without parameters.

Claim 3.5. $(V=L)$ For every set $M$ and every $X \subseteq M, H^{M}(X)$ is a $\Delta_{1}$ definable set with $M$ and $X$ as parameters.

Proof. Given $M$ and $X \subseteq M$, define a sequence $\left(H^{M}(X, n)\right)_{n<\omega}$ recursively by:

$$
\begin{aligned}
H^{M}(X, 0) & =\mathrm{Sk}^{M}{ }^{\prime}\left(\omega \times X^{<\omega}\right), \\
H^{M}(X, n+1) & =\mathrm{Sk}^{M}{ }^{\prime}\left(\omega \times H^{M}(X, n)^{<\omega}\right) .
\end{aligned}
$$

Since $\mathrm{Sk}^{M}$ is $\Delta_{1}$ definable, with $M$ as parameter, the map $n \mapsto H^{M}(X, n)$ is also $\Delta_{1}$ definable with parameters $M$ and $X$. Note that $H^{M}(X)=$ $\bigcup_{n \in \omega} H^{M}(X, n)$. Thus, for all $a$,

$$
a \in H^{M}(X) \quad \text { iff } \quad(\exists n \in \omega)\left(a \in H^{M}(x, n)\right),
$$

and so $H^{M}(X)$ is $\Delta_{1}$-definable with $M$ and $X$ as parameters.

We continue with the proof of Lemma 3.3. Recall that $T$ is a tree which is definable over $L_{\omega_{1}}$ with $\Sigma_{n}$ formulas $\varphi_{T}(x)$ and $\varphi_{\leq_{T}}(x, y)$, possibly with parameters. Then, for all $\alpha, \beta<\omega_{1}$ and every $b \in L_{\omega_{1}},\langle\alpha, \beta, b\rangle \in S_{T}$ iff $L_{\omega_{1}}$ satisfies:

(1) $\alpha$ and $\beta$ are ordinals and $\alpha<\beta$.

(2) $b$ is a function such that $(\forall \gamma \in \alpha)(\gamma \in \operatorname{dom}(b))$ and $b \in L_{\beta}$.

(3) $\left(\forall x \in L_{\beta}\right)\left(x \in H^{L_{\beta}}(\alpha \cup\{b\})\right)$ and $\left(\forall x \in H^{L_{\beta}}(\alpha \cup\{b\})\right)\left(x \in L_{\beta}\right)$.

(4) $\varphi_{T}(b\lceil\alpha)$. 
(1) is $\Delta_{0}$. Since the maps $\beta \mapsto L_{\beta}$, and $(X, M) \mapsto H^{M}(X)$ are $\Delta_{1},(2)$ and (3) are $\Delta_{1}$. Finally, it is clear that (4) is $\Sigma_{n}$.

Note that $\mu$, the Mostowski collapsing map, is $\Delta_{1}$. So, for all $\alpha, \beta, \gamma, \delta$ $<\omega_{1}$ and every $b, d \in L_{\omega_{1}},\langle\alpha, \beta, b\rangle \leq_{S_{T}}\langle\gamma, \delta, d\rangle$ iff $L_{\omega_{1}}$ satisfies:

(1) $\langle\alpha, \beta, b\rangle,\langle\gamma, \delta, d\rangle \in S_{T}$.

(2) $\alpha \leq \gamma$.

(3) $\left(\forall x \in L_{\beta}\right)\left(x \in \mu\left(H^{L_{\delta}}(\alpha \cup\{d\})\right)\right)$ and $\left(\forall x \in \mu\left(H^{L_{\delta}}(\alpha \cup\{d\})\right)\left(x \in L_{\beta}\right)\right.$.

(4) $\mu(d)=b$.

(1) is $\Sigma_{n}$ in $L_{\omega_{1}},(2)$ is $\Delta_{0}$, and (3) and (4) are $\Delta_{1}$ in $L_{\omega_{1}}$.

Therefore $\left\langle S_{T}, \leq_{S_{T}}\right\rangle$ is a tree which is $\Sigma_{n}$-definable over $L_{\omega_{1}}$.

It only remains to show that the relation $t \in\left(S_{T}\right)_{\alpha}$ is $\Sigma_{n}$ over $L_{\omega_{1}}$. But this is clear, since $t \in\left(S_{T}\right)_{\alpha}$ iff $t \in S_{T}$ and $t_{0}=\alpha$. This finishes the proof of Lemma 3.3 .

REMARK 3.6. Notice that the arguments above show that in $L$, if $\left(T, \leq_{T}\right)$ is a tree where both $T$ and $\leq_{T}$ are $\Sigma_{n}$-definable over $L_{\kappa}$ and, possibly, the levels of $T$ are not $\Sigma_{n}$-definable over $L_{\kappa}$, where $\kappa$ is an uncountable cardinal, then $S_{T}$ is a $\Sigma_{n}$-tree over $L_{\kappa}$. Thus, if $V=L$, then the conclusion of Lemma 2.7 can be strengthened to: every $\kappa$-tree that is $\Sigma_{n}$-definable over $L_{\kappa}$ has a cofinal branch. Hence, in Theorem 2.13 we can add the following as a further equivalence: $\kappa$ is inaccessible and every $\kappa$-tree that is $\Sigma_{n}$-definable over $V_{\kappa}$ has a cofinal branch.

Continuing now with the proof of Theorem 3.1, recall that WO is the $\Pi_{1}^{1}$ set of elements of the Baire space $\omega^{\omega}$ that code well-orderings of $\omega$. If $a \in \mathrm{WO}$, let $\|a\|$ be the order-type of the well-ordering coded by $a$ (see [6]). For $x \subseteq \omega$, let $\bar{x}$ be the element of $\omega^{\omega}$ coded by $x$, via some recursive bijection between $\mathcal{P}(\omega)$ and $\omega^{\omega}$.

LEMma 3.7. If $C$ is a Coll $_{\omega_{1}}-$ generic filter over $V$, then there is a function $\pi \in V[C]$ from WO into WO such that:

(1) For every $x \in \mathrm{WO}, \pi(x)$ is a code for the ordinal $\|x\|$.

(2) For every $x, y \in \mathrm{WO}$, if $\|x\|=\|y\|$, then $\pi(x)=\pi(y)$.

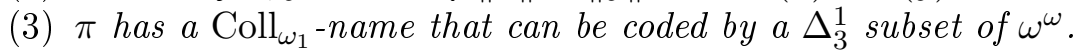

Proof. Let WO be the set of all simple Coll $\omega_{\omega_{1}}$-names $\sigma$ for a subset of $\omega$ such that $\Vdash_{\mathrm{Coll}_{\omega_{1}}}$ " $\bar{\sigma} \in \mathbf{\mathrm { WO }}$ ".

Note that, since $\operatorname{Coll}_{\omega_{1}} \in L$, every Coll $_{\omega_{1}}$-generic filter over $V$ is also generic over $L$. So, for every $\gamma<\omega_{1}$ let $\tau_{\gamma}$ be the $<_{L}$-least simple Coll $\omega_{\omega_{1}}$-name for a subset of $\omega$ such that $\Vdash_{\text {Coll }_{\omega_{1}}} "\left\|\bar{\tau}_{\gamma}\right\|=\breve{\gamma} "$. Let $B_{\omega_{1}}=\left\{\tau_{\gamma}: \gamma<\omega_{1}\right\}$ and let $\dot{B}=$ Coll $_{\omega_{1}} \times B_{\omega_{1}}$.

Define the function $\pi_{\omega_{1}}$ from WंO into $B_{\omega_{1}}$ as follows: for every $\sigma \in$ WंO, $\pi_{\omega_{1}}(\sigma)=\tau$ iff 
(1) $\tau \in B_{\omega_{1}}$,

(2) $\Vdash_{\text {Coll }_{\omega_{1}}} "\|\bar{\sigma}\|=\|\bar{\tau}\|$.

Let $\dot{\pi}=\operatorname{Coll}_{\omega_{1}} \times \pi_{\omega_{1}}$.

We can now easily check that if $C$ is $\operatorname{Coll}_{\omega_{1}}$-generic over $V$, then in $V[C]$, $\pi:=\dot{\pi}[C]$ is a function satisfying: if $\pi(a)=b$, then $\|\bar{a}\|=\|\bar{b}\|$ and $b$ is the unique code in $\dot{B}[C]$ coding the ordinal $\|\bar{a}\|$. Thus $\pi$ satisfies (1) and (2) of the lemma, modulo a recursive coding of elements of the Baire space $\omega^{\omega}$ by subsets of $\omega$.

To prove (3) we need to compute the complexity of the sets and names involved in the definition of $\pi$.

First observe that Coll $_{\omega_{1}}$ is a $\Delta_{2}^{1}$ poset (see [2]).

Let $\mathrm{WO}^{*}$ be the set of codes of elements of WO. Then $\mathrm{WO}^{*}$ is a $\Delta_{2}^{1}$ set of reals (cf. [1]).

Claim 3.8. Let $B^{*}$ be the set of all codes of elements of $B_{\omega_{1}}$. Then $B^{*}$ is a $\Delta_{3}^{1}$ set of reals.

Proof. Let $<_{L}^{*}$ be the following relation: for every $x, y \in \omega^{\omega}, x<_{L}^{*} y$ iff $x, y$ code simple Coll $_{\omega_{1}}$-names in $L$ for subsets of $\omega$ and the name coded by $x$ is $<_{L}$-less than the name coded by $y$. Since every simple Coll $\omega_{\omega_{1}}$-name for a subset of $\omega$ is hereditarily countable, the predicate " $x$ codes a simple Coll $\omega_{1}$-name in $L$ for a subset of $\omega "$ is $\Sigma_{1}$ in $H\left(\omega_{1}\right)$. Hence, as $<_{L}$ is also $\Sigma_{1}$ over $H\left(\omega_{1}\right),<_{L}^{*}$ is a $\Sigma_{2}^{1}$ relation.

Recall that $B_{\omega_{1}}$ is the range of a function that assigns to each $\gamma<\omega_{1}$ the $<_{L}$-least Coll $_{\omega_{1}}$-name for a subset of $\omega$ that is forced by Coll $\omega_{1}$ to be a code for $\gamma$. Thus, $x \in B^{*}$ iff

(1) $x$ codes a simple Coll Con $_{\omega_{1}}$-name in $L$ for a subset of $\omega$ and $\Vdash_{\text {Coll }_{\omega_{1}}}$ " $x \in$ WंO",

(2) for every $w$, if $w$ codes a simple Coll $_{\omega_{1}}$-name for a subset of $\omega$, and $w<_{L}^{*} x$, then $\nVdash_{\mathrm{Coll}_{\omega_{1}}} "\|\bar{w}\|=\|\bar{x}\| "$.

Since (1) is a $\Sigma_{2}^{1}$ sentence and (2) is $\Pi_{2}^{1}, B^{*}$ is a $\Delta_{3}^{1}$ set.

Let $\pi^{*}$ be the relation given by: $\pi^{*}(x, y)$ iff $x$ and $y$ code simple Coll $_{\omega_{1}}$ names $\sigma$ and $\tau$, respectively, for subsets of $\omega$, and $\pi_{\omega_{1}}(\sigma)=\tau$.

We will finish the proof of (3) of Lemma 3.7 by showing that $\pi^{*}$ is a $\Delta_{3}^{1}$ relation.

Let $S(v, x, y)$ iff $v$ codes a condition $p \in \operatorname{Coll}_{\omega_{1}}, x$ and $y$ code simple Coll $_{\omega_{1}}$-names $\sigma$ and $\tau$, respectively, for subsets of $\omega$, and $p \Vdash_{\mathrm{Coll}_{\omega_{1}}} "\|\bar{\sigma}\|=$ $\|\bar{\tau}\|$ ". Since the relation $\|\bar{\sigma}\|=\|\bar{\tau}\|$ is $\Sigma_{1}^{1}$, and $\operatorname{Coll}_{\omega_{1}}$ is a $\Delta_{2}^{1}$ ccc poset, $S$ is a $\Delta_{2}^{1}$ relation.

So, for every $x, y \in \omega^{\omega}, \pi^{*}(x, y)$ iff

(1) $x \in \mathrm{WO}^{*}$, 
(2) $y \in B^{*}$,

(3) $\forall v S(v, x, y)$.

Since (1) is $\Delta_{2}^{1}$, (2) is $\Delta_{3}^{1}$ and (3) is $\Pi_{2}^{1}$, we see that $\pi^{*}$ is $\Delta_{3}^{1}$. This concludes the proof of Lemma 3.7.

Recall that WF denotes the $\Pi_{1}^{1}$ set of all reals that code a well-founded relation on $\omega$ (see [6]). Every set in $H\left(\omega_{1}\right)$ can be coded by some $x \in \mathrm{WF}$ as follows: $x \in \omega^{\omega}$ codes $a \in H\left(\omega_{1}\right)$ iff $\left\langle\omega, E_{x}\right\rangle \cong\langle\mathrm{TC}(a), \in\rangle$, where for $n, m \in \omega, n E_{x} m$ iff $x(J(n, m))=0$, where $J$ is some recursive one-to-one pairing function from $\omega \times \omega$ onto $\omega$. Moreover, every $x \in \mathrm{WF}$ codes one and only one set in $H\left(\omega_{1}\right)$. So, given $x \in \mathrm{WF}$, denote by $[x]$ the set coded by $x$. Note that the map $x \mapsto[x]$ is $\Delta_{1}$ over $H\left(\omega_{1}\right)$. Let $[x] \sim[y]$ iff $x \notin$ WF or $y \notin \mathrm{WF}$ or $\left\langle\omega, E_{x}\right\rangle \cong\left\langle\omega, E_{y}\right\rangle$. Thus, $[x] \sim[y]$ is a $\Sigma_{1}^{1}$ relation on the reals. Hence, we may code every function $f \in H\left(\omega_{1}\right)$ by a real so that the set $F$ of all such codes is a $\Delta_{2}^{1}$ set of reals: for every $x \in \omega^{\omega}, x \in F$ iff

(1) $x$ codes $\left\langle x_{n}: n \in \omega\right\rangle$,

(2) $\forall n\left(x_{n}\right.$ codes $\left.\left\langle x_{n}^{0}, x_{n}^{1}\right\rangle \wedge x_{n}^{0}, x_{n}^{1} \in \mathrm{WF}\right)$,

(3) $\forall n, m\left(\left[x_{n}^{0}\right] \sim\left[x_{m}^{0}\right] \rightarrow\left[x_{n}^{1}\right] \sim\left[x_{m}^{1}\right]\right)$.

Back to the proof of Theorem 3.1, recall that we have a tree $T$ whose nodes are functions in $2^{<\omega_{1}}$ and which is $\Sigma_{1}$-definable in $L_{\omega_{1}}$. By Lemma 3.3, $S_{T}$ is also $\Sigma_{1}$-definable in $L_{\omega_{1}}$. And by Lemma $3.2, S_{T}$ is still an Aronszajn tree in $V$, and in any generic extension of $V$ that preserves $\omega_{1}$. Force with Coll $_{\omega_{1}}$ over $V$. In the generic extension $V[C]$, and using the function $\pi$ from Lemma 3.7, we may code the nodes of $S_{T}$ by reals to obtain an isomorphic tree $S_{T}^{*}$ on the reals. Namely: for all $x, y, z \in \omega^{\omega},\langle x, y, z\rangle \in S_{T}^{*}$ iff

(1) $x, y \in \mathrm{WO}$,

(2) $\pi(x)=x \wedge \pi(y)=y$,

(3) $\exists f\left(\langle\|x\|,\|y\|, f\rangle \in S_{T} \wedge z\right.$ codes the $<_{L}$-least Coll $_{\omega_{1}}$-name $\sigma$ for a real such that $\sigma[C]$ codes $f$ ).

Thus, $S_{T}^{*}$ is $\Sigma_{1}$-definable in $H\left(\omega_{1}\right)$ with $\pi$ and $C$ as additional predicates.

We will now define a version of the specializing forcing of HarringtonShelah ([5]) which will code, using $S_{T}^{*}$, any given $\omega_{1}$-sequence of reals into a single real. So, let $X$ be a fixed sequence of reals of length $\omega_{1}$, and let $X_{\alpha}$ denote the $\alpha$ th element of $X$.

Let the forcing notion $\mathbb{P}\left(S_{T}^{*}, X\right)$ be defined as follows:

- $q \in \mathbb{P}\left(S_{T}^{*}, X\right)$ iff $q$ is a finite function from $S_{T}^{*}$ into $\mathbb{Q}$ such that

(1) $(\forall s, t \in \operatorname{dom}(q))\left(s<_{S_{T}^{*}} t \rightarrow q(s)<q(t)\right)$,

(2) $(\forall s=\langle x, y, z\rangle \in \operatorname{dom}(q))((z \operatorname{codes} \sigma \wedge \sigma[C] \operatorname{codes} f \wedge$ $\left.\operatorname{dom}(f)=\omega \cdot \alpha \wedge q(s) \in \omega) \rightarrow q(s) \in X_{\alpha}\right)$.

- $q \leq q^{\prime}$ iff $q^{\prime} \subseteq q$. 
It is clear that $\mathbb{P}\left(S_{T}^{*}, X\right)$ is $\Sigma_{1}$-definable in $H\left(\omega_{1}\right)$ with $\pi, C$, and $X$ as additional predicates. And as in [5] one can show that $\mathbb{P}\left(S_{T}^{*}, X\right)$ has the property $K$, i.e., every uncountable subset contains an uncountable subset of pairwise compatible conditions. Hence it is ccc. Forcing with $\mathbb{P}\left(S_{T}^{*}, X\right)$ adds an order-preserving and continuous function $F_{X}: S_{T}^{*} \rightarrow \mathbb{Q}$, with the property that for every $n \in \omega, n \in X_{\alpha}$ iff $F(t)=n$ for some $t \in S_{T}^{*}$ of height $\omega \cdot \alpha$. Moreover, $F_{X}$ specializes $S_{T}^{*}$, i.e., for every $a \in \mathbb{Q}, F_{X}^{-1}(a)$ is an antichain of $S_{T}^{*}$.

Now let $X^{0}=\operatorname{range}(\pi)=\left\{x \in \omega^{\omega}: \exists y(y \in \mathrm{WO} \wedge \pi(y)=x)\right\}$, ordered by $x \leq_{X^{0}} x^{\prime}$ iff $x, x^{\prime} \in X_{0} \wedge\|x\| \leq\left\|x^{\prime}\right\|$. Clearly, $\left(X^{0}, \leq_{X^{0}}\right)$ is a well-ordering of reals of order-type $\omega_{1}$. By using some fixed recursive coding of elements of $\omega^{\omega}$ by subsets of $\omega$, we may assume that $X_{\alpha}^{0} \in \mathcal{P}(\omega)$ for all $\alpha<\omega_{1}$.

We next describe a finite-support iteration of length $\omega, \Delta_{2}$-definable over $H\left(\omega_{1}\right)$, with $\pi, C$, and $X^{0}$ as additional predicates. Let $\mathbb{P}_{0}=\mathbb{P}\left(S_{T}^{*}, X^{0}\right)$. Given $\mathbb{P}_{n}$, which is $\Delta_{2}$-definable over $H\left(\omega_{1}\right)$, with $\pi, C$, and $X^{0}$ as additional predicates, we define $\mathbb{P}_{n+1}$ :

For $\beta<\omega_{1}$, let $\left(S_{T}^{*}\right)_{<\beta}$ denote the set of nodes of $S_{T}^{*}$ of height $<\beta$. Notice that the predicate $x \in\left(S_{T}^{*}\right)_{<\beta}$ is $\Sigma_{1}$ in the parameter $\beta$ over $H\left(\omega_{1}\right)$.

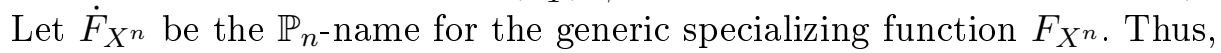

$$
\dot{F}_{X^{n}} \uparrow\left(S_{T}^{*}\right)_{<\omega \cdot(\alpha+1)}=\left\{\langle p,\langle t, r\rangle\rangle: p \in \mathbb{P}_{n},\langle t, r\rangle \in p, t \in\left(S_{T}^{*}\right)_{<\omega \cdot(\alpha+1)}\right\} .
$$

Since $\mathbb{P}_{n}$ is $\Delta_{2}$-definable over $H\left(\omega_{1}\right)$, with $\pi, C$, and $X^{0}$ as additional predicates, so is the set displayed above, with $\alpha$ as a parameter. Let $\dot{X}^{n+1}$ be a

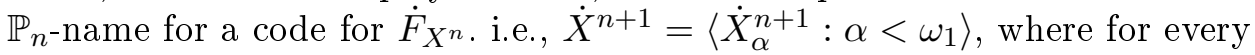
$\alpha<\omega_{1}$,

$$
\Vdash_{\mathbb{P}_{n}} " \dot{X}_{\alpha}^{n+1} \subseteq \omega \text { codes } \dot{F}_{X^{n}} \uparrow\left(S_{T}^{*}\right)_{<\omega \cdot(\alpha+1)} " .
$$

So, $\mathbb{P}_{n}$ forces that $\dot{X}_{\alpha}^{n+1}$ codes $\langle x, \dot{y}\rangle$, where $x=\left\langle x_{k}: k \in \omega\right\rangle$ codes $\left(S_{T}^{*}\right)_{<\omega \cdot(\alpha+1)}, \dot{y}=\left\langle\dot{y}_{k}: k \in \omega\right\rangle$, and $\dot{y}_{k}=\left\{\langle p, r\rangle:\left\langle x_{k}, r\right\rangle \in p\right\}$. Notice that the sentence " $x$ codes $\left(S_{T}^{*}\right)_{<\omega \cdot(\alpha+1)}$ " is $\Delta_{2}$.

Now let $\langle p, \dot{q}\rangle \in \mathbb{P}_{n+1}$ iff $p \in \mathbb{P}_{n}$ and $p \Vdash_{\mathbb{P}_{n}}$ " $\dot{q} \in \mathbb{P}\left(S_{T}^{*}, \dot{X}^{n+1}\right)$ ". Let us check that $\mathbb{P}_{n+1}$ is $\Delta_{2}$-definable over $H\left(\omega_{1}\right)$, with $\pi, C$, and $X^{0}$ as additional predicates.

First notice that the predicate " $N(\dot{q})$ iff $\dot{q}$ is a $\mathbb{P}_{n}$-name for a finite function from $S_{T}^{*}$ into $\mathbb{Q}$ " is $\Delta_{2}$. Indeed, $N(\dot{q})$ iff $\dot{q}$ is a finite set of triples $\langle q, s, r\rangle$, where $q \in \mathbb{P}_{n}, s \in S_{T}^{*}$, and $r \in \mathbb{Q}$, and for every $\left\langle q_{0}, s_{0}, r_{0}\right\rangle,\left\langle q_{1}, s_{1}, r_{1}\right\rangle \in \dot{q}$, if $s_{0}=s_{1}$ and $r_{0} \neq r_{1}$, then $q_{0} \perp q_{1}$.

Thus, we have: $p \Vdash_{\mathbb{P}_{n}}$ " $\dot{q} \in \mathbb{P}\left(S_{T}^{*}, \dot{X}^{n+1}\right)$ " iff $p \in \mathbb{P}_{n}, N(\dot{q})$, and

(1) $\forall\left\langle q_{0}, s_{0}, r_{0}\right\rangle,\left\langle q_{1}, s_{1}, r_{1}\right\rangle \in \dot{q}\left(s_{0}<_{S_{T}^{*}} s_{1} \wedge r_{1} \geq r_{0} \rightarrow q_{0} \perp q_{1}\right)$,

(2) $\forall\left\langle q_{0}, s_{0}, r_{0}\right\rangle \in \dot{q}\left(s_{0}=\langle x, y, z\rangle \wedge z\right.$ codes $\sigma \wedge \sigma[C] \operatorname{codes} f \wedge$ $\left.\operatorname{dom}(f)=\omega \cdot \alpha \wedge p \leq q_{0} \wedge r_{0} \in \omega \rightarrow q_{0} \Vdash_{\mathbb{P}_{n}} " r_{0} \in \dot{X}_{\alpha}^{n+1} "\right)$. 
But $q_{0} \Vdash_{\mathbb{P}_{n}}$ " $r_{0} \in \dot{X}_{\alpha}^{n+1}$ " iff $r_{0}=\langle k, r\rangle$ and there exists $q_{1} \leq q_{0}$ such that $\left\langle x_{k}, r\right\rangle \in q_{1}$, where $x=\left\langle x_{k}: k \in \omega\right\rangle$ is the code for $\left(S_{T}^{*}\right)_{<\omega \cdot(\alpha+1)}$.

This shows that $\mathbb{P}_{n+1}$ is also $\Delta_{2}$ over $H\left(\omega_{1}\right)$, with $\pi, C$, and $X^{0}$ as additional predicates.

Let $\mathbb{P}$ be the direct limit of the iteration $\left\langle\mathbb{P}_{n}: n<\omega\right\rangle$. Since the support of the iteration is finite, it is easily seen that $\mathbb{P}$ is $\Delta_{2}$-definable over $H\left(\omega_{1}\right)$ with $\pi, C$, and $X^{0}$ as additional predicates (see Lemma 4.1 below). Moreover, every $\mathbb{P}$-generic filter $G$ over $V[C]$ adds a real $c$ such that $X^{0} \in L[c]$ (see [5]), and so $V[C][G] \vDash " \exists x(L[x]$ has uncountably many reals)".

It is interesting to observe that $\mathbb{P}$ (and, in fact, $\left.\mathbb{P}\left(S_{T}^{*}, X\right)\right)$ is not projective in $V[C]$, as there are no uncountable projective sequences of reals in $V[C]$. However, we claim that the two-step iteration $\operatorname{Coll}_{\omega_{1}} * \mathbb{P}$ is $\Delta_{3}^{1}$.

It will be enough to show that the relation $R(x, y)$ given by:

" $x \in \operatorname{Coll}_{\omega_{1}}, y$ is a Coll $\omega_{1}$-name for a real, and $x \Vdash_{\operatorname{Coll}_{\omega_{1}}} y \in \dot{\mathbb{P}}$ "

is $\Delta_{2}$ in $H\left(\omega_{1}\right)$, without parameters.

But since $\operatorname{Coll}_{\omega_{1}}$ is a $\Delta_{2}^{1}$ forcing notion, it will be enough to see that the formula " $x \Vdash_{\operatorname{Coll}_{\omega_{1}}} y \in \dot{\mathbb{P}}$ " is equivalent both to a $\Sigma_{2}$ and a $\Pi_{2}$ formula in $H\left(\omega_{1}\right)$. For this, it is sufficient to show that the formula $y \in \dot{\mathbb{P}}$ is equivalent both to a $\Sigma_{2}$ and a $\Pi_{2}$ formula in $H\left(\omega_{1}\right)$. This is clearly so in the Coll $\omega_{1}$-name for $\pi$ as a parameter. But since by Lemma 3.7, $\pi$ has a $\operatorname{Coll}_{\omega_{1}}$-name that is $\Delta_{2}$-definable in $H\left(\omega_{1}\right)$ without parameters, we are done.

Since " $\exists x\left(L[x]\right.$ has uncountably many reals)" is a $\Sigma_{4}^{1}$ sentence, and it holds in a Coll $_{\omega_{1}} * \mathbb{P}$-generic extension of $V$, by $\Sigma_{4}^{1}$-absoluteness for $\Delta_{3}^{1}$ ccc posets, it holds in $V$. Therefore, there exists a real $x \in V$ such that $\omega_{1}^{L[x]}=\omega_{1}$, contradicting the fact that $\omega_{1}$ is inaccessible to reals. This finishes the proof of 3.1 .

Theorem 3.1 can be easily generalized:

Corollary 3.9. Let $n \geq 2$. If $\sum_{4}^{1}$ absoluteness holds for $\sum_{n+1}^{1}$ ccc forcing notions, then $\omega_{1}$ is a $\Sigma_{n}-w . c$. cardinal in $L$.

Proof. As in Theorem 3.1, if $\omega_{1}$ is not a $\Sigma_{n}$-w.c. cardinal in $L$, then there exists an Aronszajn tree $T$ on $2^{<\omega_{1}}$ which is a $\Sigma_{n}$-tree over $L_{\omega_{1}}$. As in Lemmas 3.2 and 3.3, we can find $S_{T}$, a version of the Silver tree for $T$, which is an Aronszajn tree definable over $L_{\omega_{1}}$ and has the same complexity as $T$. Using $S_{T}$, we may define the poset $\mathbb{P}$ as in Theorem 3.1 in such a way that $\operatorname{Coll}_{\omega_{1}} * \mathbb{P}$ is a $\sum_{n+1}^{1}$ and ccc poset that adds a real $x$ such that $\omega_{1}=\omega_{1}^{L[x]}$, yielding a contradiction.

We finish with two corollaries that summarize our results: 
Corollary 3.10. For every $n \geq 2$, the following are equiconsistent:

(1) $L(\mathbb{R})$-absoluteness under $\sum_{n+1}^{1}$ ccc posets.

(2) There exists a $\Sigma_{n}$-w.c. cardinal.

Corollary 3.11. The following are equiconsistent:

(1) $L(\mathbb{R})$-absoluteness under projective ccc posets.

(2) There exists a $\Sigma_{\omega}$-w.c. cardinal.

4. On iterations of projective ccc posets. We will show that after the Levy collapse of a $\Sigma_{n}$-w.c. cardinal, the property of $L(\mathbb{R})$ being a $\Sigma_{n}$-w.c. Solovay model is preserved under finite-support iterations of $\sum_{n+1}^{1}$ ccc forcing notions.

Recall that if $\mathbb{P}$ is a forcing notion, a simple $\mathbb{P}$-name for a real, i.e., for a function from $\omega$ to $\omega$, is a set $\tau$ of triples $\langle p, m, n\rangle$ such that $p \in \mathbb{P}, n, m \in \omega$, and for every $m$, the set of all $p$ such that $\langle p, m, n\rangle \in \tau$ for some $n \in \omega$, is a maximal antichain of $\mathbb{P}$.

Observe that if $\mathbb{P}$ is ccc and its conditions are real numbers, then for every simple $\mathbb{P}$-name $\tau$ for a real, $|\mathrm{TC}(\tau)|$ is countable. Further, if $\mathbb{P}$ is a finite-support iteration of ccc forcing notions whose conditions are reals, then it can be easily shown, by induction on the length of the iteration, that every simple $\mathbb{P}$-name for a real has countable transitive closure.

Lemma 4.1. Let $n \geq 1$. Suppose $L(\mathbb{R})^{M}$ is a $\Sigma_{n}$-w.c. Solovay model over $V$ and $\mathbb{P} \in M$ is the direct limit of an iteration $\left\langle\mathbb{P}_{\alpha}, \dot{\mathbb{Q}}_{\alpha}: \alpha<\lambda\right\rangle$ of countable length and with finite support such that for every $\alpha<\lambda$,

$$
\Vdash_{\mathbb{P}_{\alpha}} \text { “这 }{ }_{\alpha} \text { is a } \sum_{n+1}^{1} \text { ccc poset”. }
$$

Then the $L(\mathbb{R})$ of any $\mathbb{P}$-extension of $M$ is also a $\Sigma_{n}$-w.c. Solovay model over $V$.

Proof. Let $\kappa=\omega_{1}^{M}$. Force over $M$ to obtain a Coll $\kappa_{\kappa}$ generic $C$ over $V$ with $\mathbb{R}^{M}=\mathbb{R}^{V[C]}$ (see Lemma 1.1).

In $M$, for each $\alpha<\lambda$, fix a simple $\mathbb{P}_{\alpha}$-name $\tau_{\alpha}$ for a real that codes the parameters in some fixed $\Sigma_{n+1}^{1}$ definition of $\dot{\mathbb{Q}}_{\alpha}$.

Since the iteration is of countable length and ccc, all the $\tau_{\alpha}, \alpha<\lambda$, belong to $V[C]$ and $\mathbb{P}=\mathbb{P}^{V[C]}$, where $\mathbb{P}^{V[C]}$ is the iteration in $V[C]$ defined in the same way as $\mathbb{P}$ is defined in $M$. Moreover, a filter $G \subseteq \mathbb{P}$ is $\mathbb{P}$-generic

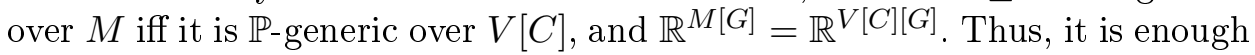
to show that for every real $x$ in $V[C][G]$ and every $X \subseteq$ Coll $_{\kappa} * \mathbb{P}$ of size less than $\kappa$ there is a complete subposet $\mathbb{Q}$ of $\operatorname{Coll}_{\kappa} * \dot{\mathbb{P}}$ such that $\mathbb{Q}$ is countable in $V[C][G], X \subseteq \mathbb{Q}$ and $x$ is $\mathbb{Q}$-generic over $V$.

We proceed by induction on $\lambda$. So we assume that for every $\alpha<\lambda$ and every $X \subseteq \operatorname{Coll}_{\kappa} * \mathbb{P}_{\alpha}$ of size less than $\kappa$, there is a complete subposet $\mathbb{Q}$ of $\operatorname{Coll}_{\kappa} * \mathbb{P}_{\alpha}$, also of size less than $\kappa$, such that $X \subseteq \mathbb{Q}$. 
We may assume that $\lambda$ is a limit ordinal, since the successor case follows directly from the proof of Theorem 2.17.

Now fix a subset $X$ of $\operatorname{Coll}_{\kappa} * \mathbb{P}$ of size less than $\kappa$, and fix a real $x$ in $V[C][G]$. Let $\dot{x} \in V$ be a simple $\operatorname{Coll}_{\kappa} * \dot{\mathbb{P}}$-name for $x$, and let $Y=$ $\mathrm{Coll}_{\kappa} * \dot{\mathbb{P}} \cap \mathrm{TC}(\dot{x})$. Since $\mathrm{Coll}_{\kappa} * \dot{\mathbb{P}}$ is $\kappa$-cc, $Y$ has cardinality less than $\kappa$. Let $Z=X \cup Y$.

For every $\alpha<\lambda$, let $Z_{\alpha}=Z \cap \operatorname{Coll}_{\kappa} * \mathbb{P}_{\alpha}$. By inductive hypothesis, we can find a $\subseteq$-increasing chain $\left\langle\mathbb{Q}_{\alpha}: \alpha<\lambda\right\rangle$ such that $\mathbb{Q}_{\alpha}$ is a complete subposet of $\operatorname{Coll}_{\kappa} * \mathbb{P}_{\alpha}$, hence also a complete subposet of $\operatorname{Coll}_{\kappa} * \mathbb{P}$, such that $Z_{\alpha} \subseteq \mathbb{Q}_{\alpha}$ for all $\alpha<\lambda$. Let $\mathbb{Q}=\bigcup_{\alpha<\lambda} \mathbb{Q}_{\alpha}$. Since the iteration has finite support, $\mathbb{Q}$ is a complete subposet of $\operatorname{Coll}_{\kappa} * \mathbb{P}$. Moreover, $\mathbb{Q}$ has size less than $\kappa$ and $Z \subseteq \mathbb{Q}$. Furthermore, letting $H=C * G \cap \mathbb{Q}$, we have $\dot{x}[H]=\dot{x}[C * G]=x$, and so $x$ is $\mathbb{Q}$-generic over $V$.

For conciseness, in what follows we will use the notation $\mathbb{P} \ll \mathbb{Q}$ to express that $\mathbb{P}$ is a complete subposet of $\mathbb{Q}$.

THEOREM 4.2. Let $\kappa$ be a $\Sigma_{n}$-w.c. cardinal, $n \geq 1$, and let $\lambda>0$. Suppose that $\mathbb{P}=\mathbb{P}_{\lambda} \in V$ is the direct limit of an iteration $\left\langle\mathbb{P}_{\alpha}, \dot{\mathbb{Q}}_{\alpha}: \alpha<\lambda\right\rangle$ with finite support such that $\mathbb{P}_{0}=\operatorname{Coll}_{\kappa}$ and for every $\alpha<\lambda$,

$$
\Vdash_{\mathbb{P}_{\alpha}} \text { “这 } \text { is a } \sum_{n+1}^{1} \text { ccc poset”. }
$$

Then the $L(\mathbb{R})$ of any $\mathbb{P}$-generic extension of $V$ is a $\Sigma_{n}$-w.c. Solovay model over $V$.

Proof. Suppose $G$ is a $\mathbb{P}$-generic filter over $V$. Notice that $\omega_{1}^{V[G]}=\kappa$, and so $\omega_{1}^{V[G]}$ is a $\Sigma_{n}$-w.c. cardinal in $V$. We only need to prove that every real in $V[G]$ is small-generic over $V$, for then it will clearly follow that for every real $x$ in $V[G], \omega_{1}^{V[G]}$ is a $\Sigma_{n}$-w.c. cardinal in $V[x]$.

The proof is by induction on $\lambda$. So, suppose that for every $\beta<\lambda$, writing $\mathbb{P}_{\beta}$ for the iteration up to $\beta$ and letting $G_{\beta}=G \cap \mathbb{P}_{\beta}$, we find that $L(\mathbb{R})^{V\left[G_{\beta}\right]}$ is a $\Sigma_{n}$-w.c. Solovay model over $V$.

Let $\mathbb{P}^{1}=\left\langle\mathbb{P}_{\alpha}^{1}, \dot{\mathbb{Q}}_{\alpha}^{1}: \alpha<\lambda\right\rangle \in V\left[G_{0}\right]$ be the remaining part of the iteration $\left\langle\mathbb{P}_{\alpha}, \dot{\mathbb{Q}}_{\alpha}: \alpha<\lambda\right\rangle$, i.e., $\mathbb{P}_{0}^{1}=\dot{\mathbb{Q}}_{0}\left[G_{0}\right], \mathbb{P}_{n+1}^{1}=\mathbb{P}_{n}^{1} * \dot{\mathbb{Q}}_{n+1}$ for $n<\omega$, and $\mathbb{P}_{\alpha+1}^{1}=\mathbb{P}_{\alpha}^{1} * \dot{\mathbb{Q}}_{\alpha}$ for $\alpha \geq \omega$. We may assume that for every $\alpha$,

$\Vdash_{\mathbb{P}_{\alpha}^{1}}$ “迹 has a largest element $\mathbf{1} "$,

and 1 is some fixed real that does not depend on $\alpha$. Moreover, we may assume that for every $p \in \mathbb{P}^{1}$ and every $\alpha<\lambda, p(\alpha)$ is a simple $\mathbb{P}_{\alpha}^{1}$-name for a real.

In $V\left[G_{0}\right]$, for each $\alpha<\lambda, \alpha>0$, fix a simple $\mathbb{P}_{\alpha}^{1}$-name $\tau_{\alpha}$ for a real that codes the parameters in a fixed $\Sigma_{n+1}^{1}$ definition of $\dot{\mathbb{Q}}_{\alpha}^{1}$, so that for some $\Sigma_{n+1}^{1}$ 
formulas $\varphi_{\alpha}(x, y), \psi_{\alpha}(x, y, z)$, and $\theta_{\alpha}(x, y, z)$,

$$
\begin{aligned}
& \Vdash_{\mathbb{P}_{\alpha}}{ } \dot{\mathbb{Q}}_{\alpha}=\left\{x: \varphi_{\alpha}\left(x, \tau_{\alpha}\right)\right\}^{\prime \prime}, \\
& \Vdash_{\mathbb{P}_{\alpha}} " \leq_{\dot{\mathbb{Q}}_{\alpha}^{1}}=\left\{\langle x, y\rangle: \psi_{\alpha}\left(x, y, \tau_{\alpha}\right)\right\} ", \\
& \Vdash_{\mathbb{P}_{\alpha}} " \perp_{\dot{\mathbb{Q}}_{\alpha}^{1}}=\left\{\langle x, y\rangle: \theta_{\alpha}\left(x, y, \tau_{\alpha}\right)\right\} " .
\end{aligned}
$$

Let $x$ be a real in $V[G]$ and let $\dot{x} \in V\left[G_{0}\right]$ be a simple $\mathbb{P}^{1}$-name for $x$.

Work in $V\left[G_{0}\right]$. Since $\mathbb{P}^{1}$ is ccc, $|\mathrm{TC}(\dot{x})|$ is countable. Let $\mu$ be a large enough regular cardinal, and let $N \preccurlyeq H(\mu)$ be such that:

(1) $\mathbb{P}^{1},\left\langle\tau_{\alpha}: \alpha<\lambda\right\rangle, \dot{x} \in N$,

(2) $\mathrm{TC}(\dot{x}) \subseteq N$,

(3) $|N|=\aleph_{0}$.

Notice that if $\alpha \in \mathrm{OR} \cap N$, then $\tau_{\alpha} \in N$, and since $\left|\mathrm{TC}\left(\tau_{\alpha}\right)\right|$ is countable, $\mathrm{TC}\left(\tau_{\alpha}\right) \subseteq N$

Now let $\mathbb{P}^{*}$ be the direct limit of the finite-support iteration $\left\langle\mathbb{P}_{\alpha}^{*}, \dot{\mathbb{Q}}_{\alpha}^{*}\right.$ : $\alpha<\lambda\rangle$ defined as follows: $\mathbb{P}_{0}^{*}=\mathbb{P}_{0}^{1}$, and $\vdash_{\mathbb{P}_{\alpha}^{*}} " \dot{\mathbb{Q}}_{\alpha}^{*}=\left\{x: \varphi_{\alpha}\left(x, \tau_{\alpha}\right)\right\} "$ if $\alpha \in \mathrm{OR} \cap N$, and $\vdash_{\mathbb{P}_{\alpha}^{*}}$ “这* $=\{\mathbf{1}\}$ ” otherwise, i.e., $\dot{\mathbb{Q}}_{\alpha}^{*}$ is the trivial poset.

We need to check that the iteration is well-defined, i.e., if $\vdash_{\mathbb{P}_{\alpha}^{*}}$ " $\dot{\mathbb{Q}}_{\alpha}^{*}=$ $\left\{x: \varphi_{\alpha}\left(x, \tau_{\alpha}\right)\right\} "$, then $\tau_{\alpha}$ is a $\mathbb{P}_{\alpha}^{*}$-name. We will show much more:

Claim 4.3.

(1) If $p \in \mathbb{P}_{\alpha}^{*}$, then $p \in \mathbb{P}_{\alpha}^{1}$. And if $p \in N$, then the converse also holds.

(2) If $\sigma$ is a simple $\mathbb{P}_{\alpha}^{*}$-name for a real, then it is also a simple $\mathbb{P}_{\alpha}^{1}$-name for a real. And if $\sigma \in N$, then the converse also holds.

(3) If $p \in \mathbb{P}_{\alpha}^{*}$ and $\sigma, \sigma^{\prime}, \tau_{\alpha}$ are simple $\mathbb{P}_{\alpha}^{*}$-names for reals, then:

(a) If $p \Vdash_{\mathbb{P}_{\alpha}^{*}} \varphi_{\alpha}\left(\sigma, \tau_{\alpha}\right)$, then $p \Vdash_{\mathbb{P}_{\alpha}^{1}} \varphi_{\alpha}\left(\sigma, \tau_{\alpha}\right)$.

(b) If $p \Vdash_{\mathbb{P}_{\alpha}^{*}} \psi_{\alpha}\left(\sigma, \sigma^{\prime}, \tau_{\alpha}\right)$, then $p \Vdash_{\mathbb{P}_{\alpha}^{1}} \psi_{\alpha}\left(\sigma, \sigma^{\prime}, \tau_{\alpha}\right)$.

(c) If $p \Vdash_{\mathbb{P}_{\alpha}^{*}} \theta_{\alpha}\left(\sigma, \sigma^{\prime}, \tau_{\alpha}\right)$, then $p \Vdash_{\mathbb{P}_{\alpha}^{1}} \theta_{\alpha}\left(\sigma, \sigma^{\prime}, \tau_{\alpha}\right)$.

And if $\alpha, p, \sigma, \sigma^{\prime} \in N$, then the converses of (a), (b), and (c) also hold.

(4) $\mathbb{P}_{\alpha}^{*} \lessdot \mathbb{P}_{\alpha}^{1}$.

Proof. By induction on $\alpha$. For $\alpha=0$ it is clear. So, let $\alpha=\beta+1$.

(1) Fix $p \in \mathbb{P}_{\alpha}^{*}$. Then $p=\left\langle p\left\lceil\beta, \sigma^{\prime}\right\rangle\right.$, where $p\left\lceil\beta \in \mathbb{P}_{\beta}^{*}, \sigma^{\prime}\right.$ is a simple $\mathbb{P}_{\beta^{-}}$ name, and either $p\left\lceil\beta \Vdash_{\mathbb{P}_{\beta}^{*}}\right.$ “ $\sigma^{\prime}=\mathbf{1}$ ", or $p\left\lceil\beta \Vdash_{\mathbb{P}_{\beta}^{*}} \varphi_{\beta}\left(\sigma^{\prime}, \tau_{\beta}\right)\right.$. So, by induction hypothesis on (1), (2), and (3)(a), we deduce that $p\left\lceil\beta \in \mathbb{P}_{\beta}^{1}, \sigma^{\prime}\right.$ is a simple $\mathbb{P}_{\beta}^{1}$-name, and either $p\left\lceil\beta \Vdash_{\mathbb{P}_{\beta}^{1}}\right.$ " $\sigma^{\prime}=\mathbf{1} "$, or $p\left\lceil\beta \Vdash_{\mathbb{P}_{\beta}^{1}} \varphi_{\beta}\left(\sigma^{\prime}, \tau_{\beta}\right)\right.$. This shows that $p \in \mathbb{P}_{\alpha}^{1}$.

Fix now $p=\left\langle p\left\lceil\beta, \sigma^{\prime}\right\rangle \in \mathbb{P}_{\alpha}^{1} \cap N\right.$. Thus, $p\left\lceil\beta \in \mathbb{P}_{\beta}^{1}, \sigma^{\prime}\right.$ is a simple $\mathbb{P}_{\beta}^{1}$-name, and $p\left\lceil\beta \Vdash_{\mathbb{P}_{\beta}^{1}} \varphi_{\beta}\left(\sigma^{\prime}, \tau_{\beta}\right)\right.$. Since $p \in N$, we also know that $p\left\lceil\beta, \sigma^{\prime} \in N\right.$. So, 
again by induction hypothesis on (1), (2), and (3)(a), we infer that $p\left\lceil\beta \in \mathbb{P}_{\beta}^{*}\right.$, $\sigma^{\prime}$ is a simple $\mathbb{P}_{\beta}^{*}$-name, and $p\left\lceil\beta \vdash_{\mathbb{P}_{\beta}^{*}} \varphi_{\beta}\left(\sigma^{\prime}, \tau_{\beta}\right)\right.$, which shows that $p \in \mathbb{P}_{\alpha}^{*}$.

(2) Now suppose that $\sigma$ is a simple $\mathbb{P}_{\alpha}^{*}$-name for a real. If $q \in \mathbb{P}_{\alpha}^{*} \cap \mathrm{TC}(\sigma)$, we can conclude as before in the case of $p$ that $q \in \mathbb{P}_{\alpha}^{1}$. This implies that $\sigma$ is a simple $\mathbb{P}_{\alpha}^{1}$-name.

If $\sigma$ is a simple $\mathbb{P}_{\alpha}^{1}$-name for a real and $\sigma \in N$, then $\operatorname{TC}(\sigma) \subseteq N$. So, if $q \in \mathbb{P}_{\alpha}^{1} \cap \mathrm{TC}(\sigma)$, we can conclude as before in the case of $p$ that $q \in \mathbb{P}_{\alpha}^{*}$. This implies that $\sigma$ is a simple $\mathbb{P}_{\alpha}^{*}$-name. In particular, if $\alpha \in N$, then $\tau_{\alpha}$ is a $\mathbb{P}_{\alpha}^{*}$-name.

(3) Suppose now that $p \in \mathbb{P}_{\alpha}^{*}, \sigma, \tau_{\alpha}$ are simple $\mathbb{P}_{\alpha}^{*}$-names for reals, and $p \Vdash_{\mathbb{P}_{\alpha}^{*}} \varphi_{\alpha}\left(\sigma, \tau_{\alpha}\right)$. We have already shown that $p \in \mathbb{P}_{\alpha}^{1}$ and $\sigma$ is a simple $\mathbb{P}_{\alpha}^{1}$-name. Since by the induction hypothesis of the theorem, $L(\mathbb{R})^{V\left[G_{0}\right]^{\mathbb{P}_{\beta}^{*}}}$ and $L(\mathbb{R})^{V\left[G_{0}\right]^{\mathbb{P}_{\beta}^{1}}}$ are both $\Sigma_{n}$-w.c. Solovay models over $V$, with the same $\omega_{1}$, and since, by induction hypothesis on $(4), \mathbb{P}_{\beta}^{*} \lessdot \mathbb{P}_{\beta}^{1}$, we also have $\mathbb{R}^{V\left[G_{0}\right]^{\mathbb{P}_{\beta}^{*}}} \subseteq \mathbb{R}^{V\left[G_{0}\right]^{\mathbb{P}_{\beta}^{1}}}$. So, by Lemma 1.3 , there exists a canonical embedding from $L(\mathbb{R})^{V\left[G_{0}\right]^{\mathbb{P}_{\beta}^{*}}}$ into $L(\mathbb{R})^{V\left[G_{0}\right]^{\mathbb{P}_{\beta}^{1}}}$. We claim that $p \Vdash_{\mathbb{P}_{\alpha}^{1}} \varphi_{\alpha}\left(\sigma, \tau_{\alpha}\right)$. Indeed, suppose $G_{\alpha}^{1}=G_{\beta}^{1} * \dot{H}$ is $\mathbb{P}_{\alpha}^{1}$-generic over $V\left[G_{0}\right]$, with $p=p\left\lceil\beta * \dot{q} \in G_{\alpha}^{1}\right.$. Since $p \Vdash_{\mathbb{P}_{\alpha}^{*}} \varphi_{\alpha}\left(\sigma, \tau_{\alpha}\right)$, and $\mathbb{P}_{\beta}^{*} \lessdot \mathbb{P}_{\beta}^{1}$, we deduce that $G_{\beta}^{*}:=G_{\beta}^{1} \cap \mathbb{P}_{\beta}^{*}$ is $\mathbb{P}_{\beta}^{*}$-generic over $V\left[G_{0}\right]$ with $p\left\lceil\beta \in G_{\beta}^{*}\right.$. Hence,

$$
V\left[G_{0}\right]\left[G_{\beta}^{*}\right] \models " i_{G_{\beta}^{*}}(\dot{q}) \Vdash_{\mathbb{Q}_{\beta}^{*}} \varphi_{\alpha}\left(\sigma, \tau_{\alpha}\right) " .
$$

Since we have $i_{G_{\beta}^{1}}(\dot{q})=i_{G_{\beta}^{*}}(\dot{q})$, by the canonical elementary embedding of $L(\mathbb{R})^{V\left[G_{0}\right]\left[G_{\beta}^{*}\right]}$ into $L(\mathbb{R})^{V\left[G_{0}\right]\left[G_{\beta}^{1}\right]}$, we obtain

$$
V\left[G_{0}\right]\left[G_{\beta}^{1}\right] \models " i_{G_{\beta}^{1}}(\dot{q}) \Vdash_{\mathbb{Q}_{\beta}^{1}} \varphi_{\alpha}\left(\sigma, \tau_{\alpha}\right) " .
$$

Hence, $V\left[G_{0}\right]\left[G_{\alpha}^{1}\right] \models \varphi_{\alpha}\left(\sigma, \tau_{\alpha}\right)$. This proves (a), and similar arguments prove (b) and (c).

Suppose now that $\alpha, p, \sigma \in N$, and $p \Vdash_{\mathbb{P}_{\alpha}^{1}} \varphi_{\alpha}\left(\sigma, \tau_{\alpha}\right)$. We have already shown that $p \in \mathbb{P}_{\alpha}^{*}$ and $\sigma, \tau_{\alpha}$ are $\mathbb{P}_{\alpha}^{*}$-names. To see that $p \Vdash_{\mathbb{P}_{\alpha}^{*}} \varphi_{\alpha}\left(\sigma, \tau_{\alpha}\right)$, suppose $G_{\alpha}^{*}=G_{\beta}^{*} * \dot{H}$ is $\mathbb{P}_{\alpha}^{*}$-generic over $V\left[G_{0}\right]$ with $p=p\left\lceil\beta * \dot{q} \in G_{\alpha}^{*}\right.$. Since $\mathbb{P}_{\beta}^{*} \lessdot \mathbb{P}_{\beta}^{1}$, we can extend $G_{\beta}^{*}$ to a $\mathbb{P}_{\beta}^{1}$-generic filter $G_{\beta}^{1}$ over $V\left[G_{0}\right]$ such that

$$
V\left[G_{0}\right]\left[G_{\beta}^{1}\right] \models " i_{G_{\beta}^{1}}(\dot{q}) \Vdash_{\mathbb{Q}_{\beta}^{1}} \varphi_{\alpha}\left(\sigma, \tau_{\alpha}\right) " .
$$

Since $i_{G_{\beta}^{1}}(\dot{q})=i_{G_{\beta}^{*}}(\dot{q})$ and since $\beta \in N$, by the canonical elementary embedding we have

$$
V\left[G_{0}\right]\left[G_{\beta}^{*}\right] \models{ }^{G_{\beta}^{*}}(\dot{q}) \Vdash_{\mathbb{Q}_{\beta}^{*}} \varphi_{\alpha}\left(\sigma, \tau_{\alpha}\right) " .
$$

Hence, $V\left[G_{0}\right]\left[G_{\alpha}^{*}\right] \models \varphi_{\alpha}\left(\sigma, \tau_{\alpha}\right)$. This proves the converse of (a), and similar arguments prove the converses of (b) and (c). 
(4) Finally, suppose $\mathbb{P}_{\beta}^{*} \lessdot \mathbb{P}_{\beta}^{1}$. By (3), $\mathbb{P}_{\alpha}^{*}$ is a subposet of $\mathbb{P}_{\alpha}^{1}$ and the incompatibility relation is preserved. Now suppose $A \in V\left[G_{0}\right]$ is a maximal antichain of $\mathbb{P}_{\alpha}^{*}$. Then $A\left\lceil\beta:=\left\{p\lceil\beta: p \in A\}\right.\right.$ is a maximal antichain of $\mathbb{P}_{\beta}^{*}$ and, by induction hypothesis, it is also a maximal antichain of $\mathbb{P}_{\beta}^{1}$. If $\beta \notin N$, then clearly $A$ is maximal in $\mathbb{P}_{\alpha}^{1}$. So, suppose $\beta \in N$. Then every $p \in A$ is of the form $\left\langle p\lceil\beta, \sigma\rangle\right.$, where $p\left\lceil\beta \Vdash_{\mathbb{Q}_{\beta}^{*}} \varphi_{\beta}\left(\sigma, \tau_{\beta}\right)\right.$. Let $A(\beta):=\{p(\beta): p \in A\}$. Then $\Vdash_{\mathbb{P}_{\beta}^{*}}$ " $A(\beta)$ is a maximal antichain of $\dot{\mathbb{Q}}_{\beta}^{*}$ ". Notice that, since $\vdash_{\mathbb{P}_{\beta}^{*}}$ " $A(\beta)$ is countable", $A(\beta) \in L(\mathbb{R})^{V\left[G_{0}\right]^{\mathbb{P}_{\beta}^{*}}}$. Thus, by the canonical embedding from $L(\mathbb{R})^{V\left[G_{0}\right]^{\mathbb{P}_{\beta}^{*}}}$ into $L(\mathbb{R})^{V\left[G_{0}\right]^{\mathbb{P}_{\beta}^{1}}}$, we conclude that $\Vdash_{\mathbb{P}_{\beta}^{1}}$ “ $A(\beta)$ is a maximal antichain of $\dot{\mathbb{Q}}_{\beta}^{1}$ ".

If $\alpha$ is a limit ordinal, then the claim follows by induction, using the fact that the iterations have finite support. This finishes the proof of the claim.

Since the iterations have finite support, it follows from the claim above that $\mathbb{P}^{*} \lessdot \mathbb{P}$. Moreover, since $\dot{x} \in N, \dot{x}$ is a $\mathbb{P}^{*}$-name. Notice that $\mathbb{P}^{*}$ is a ccc iteration.

Let $\overline{\mathbb{P}}=\left\langle\overline{\mathbb{P}}_{\beta}, \dot{\mathbb{Q}}_{\beta}: \beta<\operatorname{ot}(\mathrm{On} \cap N)\right\rangle$ be the iteration consisting of all non-trivial iterands of $\mathbb{P}^{*}$, i.e., $\overline{\mathbb{P}}_{0}=\mathbb{P}_{0}^{*}$ and for every $\beta<\operatorname{ot}(\mathrm{On} \cap N)$, $\Vdash_{\overline{\mathbb{P}}_{\beta}} \cdot \dot{\mathbb{Q}}_{\beta}=\left\{x: \varphi_{\alpha}\left(x, \tau_{\alpha}\right)\right\}$ ", where $\alpha \in N$ and $\beta=\operatorname{ot}(\alpha \cap N)$. For each $p \in \mathbb{P}^{*}$, let $\bar{p} \in \overline{\mathbb{P}}$ be the result of deleting the coordinates of $p$ that correspond to the trivial iterands of $\mathbb{P}^{*}$. Clearly, the map $e: \bar{p} \mapsto p$ is a dense complete embedding of $\overline{\mathbb{P}}$ into $\mathbb{P}^{*}$. Notice that $\dot{x}$ is a $\overline{\mathbb{P}}$-name.

Recall that $G$ is $\mathbb{P}$-generic over $V$, and $x$ is a real in $V[G]$. Let us write $G$ as $G_{0} * G^{1}$, where $G_{0}$ is $\mathbb{P}_{0}$-generic over $V$ and $G^{1}$ is $\mathbb{P}^{1}$-generic over $V\left[G_{0}\right]$. Then $\dot{x}$ is a $\mathbb{P}^{1}$-name in $V\left[G_{0}\right]$ and $i_{G^{1}}(\dot{x})=x$. Let $g=e^{-1}\left[G^{1} \cap \mathbb{P}^{*}\right]$. Then $g$ is $\overline{\mathbb{P}}$-generic over $V\left[G_{0}\right]$ and $i_{g}(\dot{x})=x$. This shows that $x$ belongs to a countable finite-support iteration over $V\left[G_{0}\right]$ of $\underset{\sim}{\sum_{n+1}^{1}}$ ccc forcing notions. So, by Lemma 4.1, $x$ is small-generic over $V$. This proves the theorem.

Corollary 4.4. Suppose that $L(\mathbb{R})^{M}$ is a $\Sigma_{\omega}$-w.c. Solovay model over $V$ and $\mathbb{P} \in M$ is the direct limit of an iteration $\left\langle\mathbb{P}_{\alpha}, \dot{\mathbb{Q}}_{\alpha}: \alpha<\lambda\right\rangle$ with finite support such that for every $\alpha<\lambda$,

$$
\Vdash_{\alpha} \text { "这 } \alpha \text { is a projective ccc poset". }
$$

Then the $L(\mathbb{R})$ of any $\mathbb{P}$-generic extension of $M$ is also a $\Sigma_{\omega}$-w.c. Solovay model over $V$.

4.1. Two applications to Martin's Axiom for projective posets. The first application will show, modulo the consistency of definable weakly compact cardinals, that Martin's Axiom restricted to posets in a given projective point-class does not imply Martin's Axiom for posets in higher point-classes. 
Definition 4.5. Let $\Gamma$ be a class of posets. Martin's Axiom for $\Gamma$, henceforth denoted by $\operatorname{MA}(\Gamma)$, is the following statement:

For every ccc poset $\mathbb{P} \in \Gamma$ and for every family $\left\langle A_{i}: i<\kappa\right\rangle, \kappa<2^{\aleph_{0}}$, of maximal antichains of $\mathbb{P}$, there exists $G \subseteq \mathbb{P}$ directed such that for every $i<\kappa, G \cap A_{i} \neq \emptyset$.

For every $n \geq 1, \mathrm{MA}\left(\sum_{n}^{1}\right)$ is Martin's Axiom for $\Sigma_{n}^{1}$ posets. MA(Proj) is Martin's Axiom for projective posets.

THEOREM 4.6. Let $n \geq 1$, and suppose that there exists a $\Sigma_{n}$-w.c. cardinal in $L$. Then there exists a poset $\mathbb{P}$ such that for every $\mathbb{P}$-generic filter $G$ over $L$,

$$
L[G] \models \mathrm{MA}\left(\sum_{n+1}^{1}\right) \wedge \neg \mathrm{MA}\left(\Sigma_{n+2}^{1}\right) .
$$

Proof. Let $\kappa$ be the least $\Sigma_{n}$-w.c. cardinal in $L$. Let $\mathbb{P}$ be the direct limit of an iteration $\left\langle\mathbb{P}_{\alpha}, \dot{\mathbb{Q}}_{\alpha}: \alpha<\kappa^{+}\right\rangle$, with finite support, where $\mathbb{P}_{0}=$ Coll $_{\kappa}$ and for every $\alpha<\kappa^{+}$,

$$
\Vdash_{\alpha} \text { " } \dot{Q}_{\alpha} \text { is a } \sum_{n+1}^{1} \text { ccc forcing notion", }
$$

so that for every $\mathbb{P}$-generic filter $G$ over $L$,

$$
L[G] \models \operatorname{MA}\left(\sum_{n+1}^{1}\right) \wedge 2^{\aleph_{0}}=\aleph_{2}
$$

(see [1, Theorem 3.10]).

Now assume, towards a contradiction, that

$$
L[G] \models \operatorname{MA}\left(\Sigma_{n+2}^{1}\right) .
$$

Then, since $\omega_{1}^{L[G]}=\kappa$ is not a $\Sigma_{n+1}$-w.c. cardinal in $L$, there is, in $L$, a $\kappa$-Aronszajn tree $T$ which is $\Sigma_{n+1}$-definable over $L_{\kappa}$. As in the proof of Theorem 3.1 we may define a $\Sigma_{n+2}^{1}$ ccc poset of the form $\operatorname{Coll}_{\omega_{1}} * \mathbb{P}$ such that $\operatorname{MA}\left(\operatorname{Coll}_{\omega_{1}} * \mathbb{P}\right)$ implies that there exists a real $x$ such that $\omega_{1}^{L[G]}=\omega_{1}^{L[x]}$. But then $L(\mathbb{R})^{L[G]}$ is not a $\Sigma_{n}$-w.c. Solovay model over $V$, in contradiction with Theorem 4.2.

COROLlary 4.7. Let $n \geq 1$ and suppose that the existence of a $\Sigma_{n}-w . c$. cardinal is consistent with $\mathrm{ZFC}$. Then $\mathrm{ZFC}+\mathrm{MA}\left(\sum_{n+1}^{1}\right)$ does not imply $\operatorname{MA}\left(\Sigma_{n+2}^{1}\right)$.

It is known that if $\mathrm{ZFC}$ is consistent, then $\mathrm{ZFC}+\mathrm{MA}\left(\Sigma_{1}^{1}\right)$ does not imply $\operatorname{MA}\left(\Sigma_{2}^{1}\right)$ (see [1, Section 5]).

For the second application, let $\varphi$ be the statement "Every set of reals in $L(\mathbb{R})$ is Lebesgue measurable, has the property of Baire, is Ramsey, and has the perfect set property". 
THEOREM 4.8. Let $n \geq 1$, and suppose that there exists a $\Sigma_{n}$-w.c. cardinal. Then there exists a poset $\mathbb{P}$ such that for every $\mathbb{P}$-generic filter $G$ over $V$,

$$
V[G] \models \mathrm{MA}\left(\sum_{n+1}^{1}\right) \wedge \neg \mathrm{CH}+\varphi .
$$

Proof. Let $\kappa$ be a $\Sigma_{n}$-w.c. cardinal, and let $\mathbb{P}$ be the direct limit of a finite-support iteration $\left\langle\mathbb{P}_{\alpha}, \dot{\mathbb{Q}}_{\alpha}: \alpha<\kappa^{+}\right\rangle$, where $\mathbb{P}_{0}=$ Coll $_{\kappa}$ and for every $\alpha<\kappa^{+}$

$$
\Vdash_{\alpha} \text { " } \dot{Q}_{\alpha} \text { is a } \sum_{n+1}^{1} \text { ccc forcing notion", }
$$

so that for every $\mathbb{P}$-generic filter $G$ over $V$,

$$
V[G] \models \operatorname{MA}\left(\sum_{n+1}^{1}\right) \wedge 2^{\aleph_{0}}=\aleph_{2}
$$

(see [1, Theorem 3.10]). By Theorem 4.2, $L(\mathbb{R})^{V[G]}$ is a $\Sigma_{n \text {-w.c. Solovay }}$ model over $V$. Thus,

$$
V[G] \models \varphi
$$

Corollary 4.9 .

(1) For every $n \geq 1$, Con(ZFC + there exists a $\Sigma_{n}-w . c$. cardinal) implies $\mathrm{Con}\left(\mathrm{ZFC}+\mathrm{MA}\left(\sum_{n+1}^{1}\right)+\neg \mathrm{CH}+\varphi\right)$.

(2) $\mathrm{Con}\left(\mathrm{ZFC}+\right.$ there exists a $\Sigma_{\omega}$-w.c. cardinal $)$ implies $\mathrm{Con}(\mathrm{ZFC}+$ $\mathrm{MA}($ Proj $)+\neg \mathrm{CH}+\varphi)$.

\section{References}

[1] J. Bagaria and R. Bosch, Projective forcing, Ann. Pure Appl. Logic 86 (1997), 237-266.

[2] - - - Solovay models and ccc forcing extensions, J. Symbolic Logic 69 (2004), $742-766$.

[3] —, - Proper forcing extensions and Solovay models, Arch. Math. Logic 43 (2004), 739-750.

[4] R. Bosch, Small definably-large cardinals, in: J. Bagaria and S. Todorcevic (eds.), Set Theory. Centre de Ricerca Matemàtica, Barcelona, 2003-2004, Birkhäuser, 2006, $55-82$.

[5] L. Harrington and S. Shelah, Some exact equiconsistency results, Notre Dame J. Formal Logic 26 (1985), 178-188.

[6] T. Jech, Set Theory, 3rd ed., Springer, 2003.

[7] H. Judah and Andrzej Rosłanowski, Martin Axiom and the size of the continuum, J. Symbolic Logic 60 (1995), 374-391.

[8] E. Kranakis, Definable partitions and reflection properties for regular cardinals, Notre Dame J. Formal Logic 26 (1985), 408-412.

[9] A. Leshem, On the consistency of the definable tree property on $\aleph_{1}$, J. Symbolic Logic 65 (2000), 1204-1214. 
[10] R. Solovay, A model of set theory in which every set of reals is Lebesgue measurable, Ann. of Math. 92 (1970), 1-56.

ICREA (Institució Catalana

de Recerca i Estudis Avançats)

and Departament de Lògica, Història

i Filosofia de la Ciència

Universitat de Barcelona

Montalegre 6

08001 Barcelona, Spain

E-mail: bagaria@ub.edu
Departamento de Filosofía Universidad de Oviedo Teniente Alfonso Martínez, s/n 33011 Oviedo, Spain E-mail: roger@uniovi.es

Received 5 November 2005;

in revised form 21 February 2007 